\title{
Modified Dynamical Supergravity Breaking and Off-Diagonal Super-Higgs Effects
}

\author{
Tamara Gheorghiu *1, Olivia Vacaru ${ }^{\dagger 2}$, and Sergiu Vacaru ${ }^{\ddagger 3}$ \\ ${ }^{1}$ Project IDEI, Alexandru Ioan Cuza University, Alexandru Lapuşneanu street, \\ nr. 14, UAIC - Corpus R, office 323; Iaşi, Romania, 700057 \\ ${ }^{2}$ National College of Iaşi; Arcu street, nr. 4, Iaşi, Romania, 700125 \\ ${ }^{3}$ Theory Division, CERN, CH-1211, Geneva 23, Switzerland ${ }^{\S}$; and \\ Rector's Office, Alexandru Ioan Cuza University, Alexandru Lapuşneanu street, \\ nr. 14, UAIC - Corpus R, office 323; Iaşi, Romania, 700057
}

October 2, 2014

\begin{abstract}
We argue that generic off-diagonal vacuum and nonvacuum solutions for Einstein manifolds mimic physical effects in modified gravity theories (MGTs) and encode certain models of the $f(R, T, \ldots)$, Hořava type with dynamical Lorentz symmetry breaking, induced effective mass for the graviton etc. Our main goal is to investigate the dynamical breaking of local supersymmetry determined by off-diagonal solutions in MGTs and encoded as effective Einstein spaces. This includes the Deser-Zumino super-Higgs effect, for instance, for a oneloop potential in a (simple but representative) model of $\mathcal{N}=1, D=4$ supergravity. We develop and apply new geometrical techniques which allows us to decouple the gravitational field equations and integrate them in a very general form with the metric and vielbein fields depending on all the spacetime coordinates via means of various generating
\end{abstract}

*tamara.gheorghiu@yahoo.com

†olivia.vacaru@yahoo.com

${ }^{\ddagger}$ sergiu.vacaru@cern.ch; sergiu.vacaru@uaic.ro

$\S$ associated visiting researcher 
and integration functions and parameters. We study how solutions in MGTs may be related to the dynamical generation of a gravitino mass and supersymmetry breaking.

Keywords: dynamical supergravity breaking, super-Higgs effects, gravitational solitons, modified (super) gravity

PACS numbers: 98.80.-k, 04.50.Kd, 95.36. $+\mathrm{x}$

\section{Contents}

1 Introduction 3

2 Encoding MGTs into Off-Diagonal Metrics and Torsions 7

2.1 Preliminaries ..................... 7

2.1.1 N-adapted frames, $\mathrm{d}$-metrics and $\mathrm{d}$-connections . . . 7

2.1.2 Nonholonomic torsions in (pseudo) Riemannian geometry 9

2.2 Equivalent modelling of MGTs . . . . . . . . . . . . . . . . 10

2.3 The AFDM in MGT . . . . . . . . . . . . . . . . 12

2.3.1 Decoupling property for the canonical $\mathrm{d}$-connection with respect to $\mathrm{N}$-adapted frames . . . . . . . . . . 13

2.3.2 Generating off-diagonal solutions . . . . . . . . . . 14

2.3.3 Encoding MGTs as nonholonomic Einstein manifolds 15

2.3.4 Examples: off-diagonal solutions and torsion . . . . . 16

3 A $\mathcal{N}=1, d=4$ Modified Supergravity Theory 19

3.1 Nonholonomic super-Higgs effects and goldstino coupling . . . 19

3.2 An Einstein-Cartan like formulation of MSGT . . . . . . . . . 20

3.2.1 The nonholonomic supersymmetric Lagrangian . . . . . 20

3.2.2 Off-diagonal mass generation for the gravitino . . . . . 21

4 One-Loop Partition Functions with MGT Deformations 22

4.1 Nonholonomic bosonic configurations . . . . . . . . . . . . 23

4.1.1 Bosonic operators . . . . . . . . . . . . . 23

4.1.2 N-adapted gauge fixing and physical gauge . . . . . . 24

4.1.3 MGT partition function . . . . . . . . . 25

4.2 Nonholonomic fermionic configurations . . . . . . . . . . . 26

4.3 Effective MGT supersymmetry breaking . . . . . . . . . . 27

4.3.1 One-loop effective potential and stability . . . . . . 27

4.3.2 Off-diagonal super-Higgs effects and fermionic and bosonic contributions . . . . . . . . . . . . . 29

4.3.3 On super-Higgs effects for MGT solitonic configurations 30 


\section{Introduction}

Recently, the Nobel Prize in Physics 2013 was awarded to F. Englert and P. W. Higgs [1]. Their theoretical work was "confirmed" after the discovery of the predicted fundamental particle, by the ATLAS and CMS experiments at CERN's Large Hadron Collider" [2]. Related to this discovery, we note that supersymmetry remains still an important theoretical activity in the construction of various unifying models in low energy physics. For instance, in the study of the stability of the Higgs vacuum and unification of the fundamental forces, and/or the low scale of inflation. Supergravity (SG) theories are conjectured to be related to the low-energy limits of superstring theories and which are the candidates for a theory of quantum gravity and its unfication with the other fundamental interactions.

If it exists, supersymmetry must be broken at low energies because there is not any experimental data (and related phenomenological models) confirming such generalized symmetry. We cite early work [3] when the general relativity (GR) theory was obtained in the standard low energy limit of superstring and supegravity theory. Perhaps this is related to the spontaneous or dynamical symetry breaking of a fundamental theory and to various modified gravity theories (MGTs). We consider that it is desirable to elaborate a formalism of breaking the local supersymmetry directly, i.e. without the coupling to a gauge sector. A typical example is the one used for the breaking of chiral symmetry in [4. Our goal is to extend this approach when MGTs (and in particular, off-diagonal solutions in GR) are included into the scheme of dynamical breaking of local supersymmetry.

There are several important motivations for the elaboration and study of MGTs. A number of theoretical attempts have been proposed in order to explain the accelerated expansion of the universe, solve the dark energy and dark matter problems and find consistency with the observational data in modern cosmology. To formulate a self-consistent quantum gravity theory seems very difficult to achieve within the framework of the ordinary GR theory. We cite here some of most popular approaches elaborated during the last 25 years. For instance, the DGP braneworld model by Dvali, Gabadadze and Porrati [5]) is a version of the theories with warped/ trapped configurations and extra dimensions which can be extended to off-diagonal configurations, see [6]. There is certain "cosmological" equivalence between such models and some classes of Finsler-like theories [7], see papers [8] for reviews and recent results on the so-called Lagrange-Finsler modifications of gravity theories. 
Perhaps, one of the most popular class of MGTs consists of the so-called $f$-modified theories when certain functionals $f(R), f(R, T)$ of the curvature and torsion modify the standard form of the Einstein-Hilbert Lagrangian of GR. In particular, we can take $\mathcal{L}=R$, on a pseudo-Riemannian manifold, $V$, where $R$ is the Ricci scalar associated with the Levi-Civita connection $\nabla$. Other corresponding functionals depend on the torsion tensor, $T_{\beta \gamma}^{\alpha}$, the matter energy-momentum tenso, $T_{\beta \gamma}$, and/or trace $T=T_{\alpha}^{\alpha}$, on the Ricci scalar $\mathbf{R}$ for a generalized connection $\mathbf{D}$ etc. The physical/ geometric objects for such theories are defined on the tangent bundle $T V$, or on a generalized nonholonomic manifold $\mathbf{V}$ with a prescribed nonholonomic structure of frames, distortions of geometric objects etc [9]. This includes theories with Lorentz violations, nonlinear dispersion relations, locally anisotropic re-scaling and effective polarizations of constants which provides a deeper understanding of the relations among $f$-modified, Hořava-Lifshitz and Finsler-like theories.

For MGTs with nontrivial torsion, we can naturally find motivations for the presence of fermionic (gravitino) terms. Such configurations are generically present in SG theories via four-gravitino self-interaction terms which may produce condensates of the gravitino field under well-defined conditions. For certain models, the gravitino may acquire mass but leave the graviton massless. In general, we can consider bi-metric and massive theories [10] for off-diagonal configurations encoding MGTs contributions [11]. In the model [12], the mass of the gravitino is generated dynamically to be of order of the Planck scale. It was conjectured that the dynamical breaking of local supersymmetry is a result of the formation of condensates of the gravitino filed. The approach was studied for the Deser-Zumino super-Higgs effect [13] which resulted from a coupling of the supergravity action with the nonlinear Volkov-Akulov action[14] and the Goldsone particle. Assuming a F-type spontaneous global supersymmetry breaking, the final result is a Majorana spin $1 / 2$ fermion for the so-called Godstino field.

The authors of [15] criticised the flat Minkowski spacetime approach [12] because it ignored the quantum fluctuations of the metric field. Such fluctuations over the metric backgrounds introduce imaginary parts to the one-loop effective potential in four-dimensional supergravity theories for any nontrivial value of the gravitino condensate field 1 In general, this does not depend on the introduction of a possible background cosmological constant $\Lambda$ which may replace [16] the ultra violet cut-off used in the flat spacetime. Recently, the arguments of [15] were reconsidered in [17] by incorporating the super-Higgs effect with the aim to build a simple model of dy-

\footnotetext{
${ }^{1}$ Imaginary parts may result in the instability of the non-trivial-gravitino-condensate (breaking $\mathcal{N}=1$ supergravity).
} 
namical breaking of local supersymmetry by means of the gravitino-torsion self-interactions. Torsion fields exist in various (super) string and gravity theories and it is naturally to consider that they may be responsible for the local supersymmetry breaking. In another turn, various models of MGTs were studied recently for certain functional modifications of the Lagrangians with anisotropic symmetries on certain effective pseudo-Riemannian spaces (which in terms of the Levi-Civita (LC) connection are torsionless).

Our goal is to study three problems based in conjecturing that a dynamical breaking of supergravity is possible via the formation of condensates of the gravitino fields when the spacetime is not flat in GR or in MGT. The problems are : 1) Will super-Higgs generalized effects help in the construction of modified supergravity theories (MSGT)? 2) How such super-Higgs models are related to the various classes of generic off-diagonal solutions that can be constructed in GR and MGTs? 3) How a mechanism of dynamical SG-breaking can be related to an accelerating universe and to dark energy and dark matter cosmology? In this article, we shall develop the approaches taken from [15, 17] to investigate possible dynamical breaking scenarios in supergravity which may be connected with certain limits (not only of GR) but of MGTs and to study the corresponding physically important classes of solutions which can be realized as effective off-diagonal Einstein manifolds.

Let us provide certain motivations for our work. The main idea comes from a (very surprising) decoupling property of the vacuum and non-vacuum fundamental field equations in certain classes of gravity theories. In a simple way, such a property can be proven if one works with an "auxiliary" connection when certain associated systems of nonlinear PDE decouple with respect to certain classes of nonholonomic frames. Hence, we can integrate such systems in very general forms which allows us to generate and study new classes of generic off-diagonal solutions.2 Usually, an auxiliary connection has a nontrivial nonholonomically induced torsion which in GR is completely determined by certain generic off-diagonal terms in the metrics and/or after imposing certain classes of nonholonomic (equivalently, anholonomic, i.e. non-integrable) constraints on the gravitational and matter fields dynamics. We note that, in general, a nonholonomically induced torsion is different from the torsion fields in the Einstein-Cartan gauge theories of gravity, or in string gravity theory. In our approach, the torsion is induced by the off-diagonal terms of the metric field and (not obligatory) subjected to additional algebraic or dynamical field equations. The geometric formalism with nonholonomic configurations can be extended to various classes of commutative and noncommutative, or supersymmetric gravity and field theories,

\footnotetext{
${ }^{2}$ The corresponding metrics cannot be diagonalized by coordinate transformations.
} 
by relating nonholonomic frame deformations to nontrivial torsion fields of a different nature. Having constructed certain general integral varieties of solutions, we can impose almost always certain classes of constraints when the auxiliary connection transforms into the Levi-Civita (LC) one. Such zero-torsion constraints can be imposed additionally after certain classes of generalized solutions are found, i.e. generic off-diagonal solutions for nonlinear systems can be restricted to have torsionless configurations if certain nontrivial solutions were already found for the decoupled configurations. The end result is that it was possible to construct a geometric formalism, the so-called anholonomic frame deformation method (AFDM), for constructing exact solutions in gravity [6].

Our goal is to demonstrate in detailed that a nonholonomic (and alternative off-diagonal) incorporation of the super-Higgs effect may result in the dynamical breaking of MSGTs, see examples of such works and applications in cosmology [18. This can be realized prior to the coupling to matter and gauge fields. The AFDM allows us to encode various modifications of (super) gravitational theories into off-diagonal Einstein spaces whose effective cosmological constant which can be chosen to be positive for certain welldefined conditions. We can perform a one-loop effective potential calculation considering the metric fluctuations fully and (not small) off-diagonal deformations, about the de Sitter backgrounds. Within the framework of the correspondingly defined nonholonomic frames, any (weak) quantum gravitational effects reveals the existence of non-trivial vacua with no imaginary parts. In this way, we cured the problems mentioned in [15] and generalized the constructions of [17] for various classes of MGTs. The effective positive cosmological constant (which in the AFDM is a simple parameter used for the re-definition of an integrating function) acquires a physical meaning and becomes responsible for the vanishing of the effective vacuum energy. This is due to the super-Higgs effect which is determined by the one-loop low-energy effective action of supergravity.

The article is organized as follows. In Sec. 2, we survey a geometric technique for generating off-diagonal solutions with an associated torsion structure in MGT and GR. Explicit examples are considered of three-dimensional gravitational solitonic waves possessing different properties and having possible effects in supergravity and modified gravity. Sec. 3 is devoted to $\mathcal{N}=1, d=4$ modified supergravity models with nonholonomic super-Higgs effects and goldstino coupling. In Sec. 4, we perform a one-loop calculation in order to compute the partition functions in MGT deformations with nonholonomic bosonic and fermionic configurations. We discuss in brief the stability conditions determined by the nonholonomic constraints and nonlinear off-diagonal interactions and also discuss how such issues can be related 
to solitonic solutions in gravity theories. Finally, a discussion of the results and the conclusions are presented in Sec. 5.

\section{Encoding MGTs into Off-Diagonal Metrics and Torsions}

Various classes of MGTs were elaborated with the aim to explain observational data in modern cosmology and solve the dark energy and dark matter problems. In this section we outline a geometric approach which allows us to encode various classes of solutions of field equations in MGTs into the corresponding off-diagonal metrics that define the (effective) Einstein manifolds. We summarize also the anholonomic frame deformation method, AFDM, for constructing off-diagonal solutions in gravity and Ricci flow theories [6, 8]. This is possible for metric-compatible linear connections with noholonomically induced torsion and that are completely defined by the metric tensors and the anholonomy frame coefficients. After imposing additional nonintregrable constraints, we can "extract" solutions for the Levi-Civita connection $\nabla$.

\subsection{Preliminaries}

Our key geometric idea is that GR and various MGTs can be described equivalently in terms of the geometric data $(\mathbf{g}, \nabla)$ and/ or $(\mathbf{g}, \widehat{\mathbf{D}})$ using an "auxiliary" canonical distinguished connection $\widehat{\mathbf{D}}$ which also is completely defined by the same metric structure g. In general, $\widehat{\mathbf{D}}$ has a nontrivial nonholonomically induced torsion $\widehat{\mathcal{T}}$ determined by certain generic off-diagonal coefficients of $\mathbf{g} 3$ Using $\widehat{\mathcal{T}}$ and the corresponding classes of exact solutions in MGT, we find additional mechanisms for dynamical supersymmetry breaking if the constructions are extended to SG.

\subsubsection{N-adapted frames, $\mathrm{d}-$-metrics and $\mathrm{d}-$-connections}

Any metric structure $\mathbf{g}$ on a pseudo-Riemannian manifold $\mathbf{V}$, for instance, of signature $(+,+,+,-)$, can be written in two equivalent forms:

1. for a local coordinate co-base,

$$
\mathbf{g}=\underline{g}_{\alpha \beta} d u^{\alpha} \otimes d u^{\beta}
$$

\footnotetext{
${ }^{3}$ Such a metric is considered generic off-diagonal if it cannot be diagonalized via coordinate transformations.
} 
with coefficients

$$
\underline{g}_{\alpha \beta}=\left[\begin{array}{cc}
g_{i j}+N_{i}^{a} N_{j}^{b} g_{a b} & N_{j}^{e} g_{a e} \\
N_{i}^{e} g_{b e} & g_{a b}
\end{array}\right],
$$

for coordinates $u=(x, y)$, or $u^{\alpha}=\left(x^{i}, y^{a}\right)$, and $h$-indices $i, j, \ldots=$ $1,2, \ldots n$ and $v$-indices $a, b, \ldots=n+1, n+2, \ldots, n+m$; when $\operatorname{dim} \mathbf{V}=$ $n+m$, for $n, m \geq 2$ 4

2. We can represent $\mathbf{g}$ as a distinguished metric (in brief, $d$-metric) 5

$$
\mathbf{g}=g_{\alpha}(u) \mathbf{e}^{\alpha} \otimes \mathbf{e}^{\alpha}=g_{i}\left(x^{k}\right) d x^{i} \otimes d x^{i}+g_{a}\left(x^{k}, y^{b}\right) \mathbf{e}^{a} \otimes \mathbf{e}^{a} .
$$

In the $\mathrm{N}$-adapted form the nonholonomic frames $\mathbf{e}_{\nu}=\left(\mathbf{e}_{i}, e_{a}\right)$ and $\mathbf{e}^{\mu}=$ $\left(e^{i}, \mathbf{e}^{a}\right)$, are

$$
\begin{aligned}
\mathbf{e}_{i} & =\partial / \partial x^{i}-N_{i}^{a}(u) \partial / \partial y^{a}, e_{a}=\partial_{a}=\partial / \partial y^{a} \\
e^{i} & =d x^{i}, \mathbf{e}^{a}=d y^{a}+N_{i}^{a}(u) d x^{i} .
\end{aligned}
$$

They satisfy the relations

$$
\left[\mathbf{e}_{\alpha}, \mathbf{e}_{\beta}\right]=\mathbf{e}_{\alpha} \mathbf{e}_{\beta}-\mathbf{e}_{\beta} \mathbf{e}_{\alpha}=W_{\alpha \beta}^{\gamma} \mathbf{e}_{\gamma},
$$

whose anholonomy coefficients are $W_{i a}^{b}=\partial_{a} N_{i}^{b}, W_{j i}^{a}=\Omega_{i j}^{a}=\mathbf{e}_{j}\left(N_{i}^{a}\right)-$ $\mathbf{e}_{i}\left(N_{j}^{a}\right)$. In these formulas, the coefficients $\Omega_{i j}^{a}$ define the $\mathrm{N}$-connection curvature, see below.

The coefficients $\mathbf{N}=\left\{N_{i}^{a}(u)\right\}=N_{i}^{a}(x, y) d x^{i} \otimes \partial / \partial y^{a}$ define a nonholonomic (equivalently, non-integrable, or anholonomic) horizontal (h) and vertical (v) splitting, or a nonlinear connection ( $N$-connection) structure if a Whitney sum $\mathbf{N}$ is obtained for its tangent space $T \mathbf{V}$,

$$
\mathbf{N}: T \mathbf{V}=h T \mathbf{V} \oplus v T \mathbf{V} \text {. }
$$

\footnotetext{
${ }^{4}$ The Einstein summation rule on the "up-low" cross indices is used unless otherwise stated. Boldface letters shall be used in order to emphasize that a $\mathrm{N}$-connection splitting has been considered on the spacetime manifold $\mathbf{V}=(V, \mathbf{N})$. In order to avoid cumbersome formulas, we shall write off-diagonal terms in explicit form only for 4-d configurations (if this does not result in ambiguities). In general, our approach can be elaborated for arbitrary finite dimensions.

${ }^{5}$ this term is largely used in some monographs on differential geometry but also in some series of works on gravity and mathematical particle physics [6, 8, 22, 24], see also references therein; it was introduced with three main purposes: to elaborate geometric constructions adapted with respect to certain natural/ conventional h- and v-splitting which allows to consider further parameterizations in order to decouple gravitational field equations for corresponding classes of nonlinear and linear connections and, finally, to perform deformation quantization of such nonlinear systems
} 
We say that a linear connection is a distinguished connection, $d$-connection, $\mathbf{D}=(h D, v D)$, if it preserves under parallelism a $h-v$-splitting (6). It defines a covariant derivative, $\mathbf{D}_{\mathbf{X}} \mathbf{Y}$, for a d-vector field $\mathbf{Y}$ in the direction of a d-vector $\mathbf{X}$. In general, any vector field $Y(u) \in T \mathbf{V}$ can be parameterized as a d-vector, $\mathbf{Y}=\mathbf{Y}^{\alpha} \mathbf{e}_{\alpha}=\mathbf{Y}^{i} \mathbf{e}_{i}+\mathbf{Y}^{a} e_{a}$, or $\mathbf{Y}=(h Y, v Y)$, with $h Y=\left\{\mathbf{Y}^{i}\right\}$ and $v Y=\left\{\mathbf{Y}^{a}\right\}$. The local coefficients $\mathbf{D}=\left\{\boldsymbol{\Gamma}_{\alpha \beta}^{\gamma}=\left(L_{j k}^{i}, L_{b k}^{a}, C_{j c}^{i}, C_{b c}^{a}\right)\right\}$ are computed as h-v-components of $\mathbf{D}_{\mathbf{e}_{\alpha}} \mathbf{e}_{\beta}:=\mathbf{D}_{\alpha} \mathbf{e}_{\beta}$ using $\mathbf{X}=\mathbf{e}_{\alpha}$ and $\mathbf{Y}=\mathbf{e}_{\beta}$. The terms $\mathrm{d}$-vector, $\mathrm{d}$-tensor etc can be used for any vector, tensor value with coefficients defined in the $\mathrm{N}$-adapted form with respect to the necessary types of tensor products of bases (3) and (4).

For any d-connection structure $\mathbf{D}$, we can construct three fundamental geometric objects: the d-torsion, $\mathcal{T}(\mathbf{X}, \mathbf{Y}):=\mathbf{D}_{\mathbf{X}} \mathbf{Y}-\mathbf{D}_{\mathbf{Y}} \mathbf{X}-[\mathbf{X}, \mathbf{Y}]$, the nonmetricity, $\mathcal{Q}(\mathbf{X}):=\mathbf{D}_{\mathbf{X}} \mathbf{g}$, and the d-curvature, $\mathcal{R}(\mathbf{X}, \mathbf{Y}):=\mathbf{D}_{\mathbf{X}} \mathbf{D}_{\mathbf{Y}}-$ $\mathbf{D}_{\mathbf{Y}} \mathbf{D}_{\mathbf{X}}-\mathbf{D}_{[\mathbf{X}, \mathbf{Y}]}$. Respectively, the N-adapted coefficients,

$$
\begin{aligned}
& \mathcal{T}=\left\{\mathbf{T}^{\gamma}{ }_{\alpha \beta}=\left(T_{j k}^{i}, T^{i}{ }_{j a}, T_{j i}^{a}, T_{b i}^{a}, T_{b c}^{a}\right)\right\}, \mathcal{Q}=\left\{\mathbf{Q}_{\alpha \beta}^{\gamma}\right\}, \\
& \mathcal{R}=\left\{\mathbf{R}_{\beta \gamma \delta}^{\alpha}=\left(R_{h j k}^{i}, R_{b j k}^{a}, R^{i}{ }_{h j a}, R_{b j a}^{c}, R^{i}{ }_{h b a}, R_{b e a}^{c}\right)\right\}
\end{aligned}
$$

are computed by introducing $\mathbf{X}=\mathbf{e}_{\alpha}$ and $\mathbf{Y}=\mathbf{e}_{\beta}$, and $\mathbf{D}=\left\{\boldsymbol{\Gamma}_{\alpha \beta}^{\gamma}\right\}$ into above formulas, see details in Refs. [6].

A d-connection $\mathbf{D}$ is compatible with a d-metric $\mathbf{g}$ if and only if $\mathcal{Q}=$ $\mathbf{D g}=0$. The Levi-Civita (LC) connection, $\nabla$, is the unique metric compatible and torsionless linear connection determined by a metric structure $\mathbf{g}$. We can always consider a $\mathrm{N}$-adapted distortion of a $\mathrm{d}$-connection $\mathbf{D}$ when

$$
\mathbf{D}=\nabla+\mathbf{Z}
$$

The N-adapted coefficients are labeled respectively: $\nabla=\left\{\Gamma_{\beta \gamma}^{\alpha}\right\}$ and, for the distortion d-tensor, $\mathbf{Z}=\left\{\mathbf{Z}_{\beta \gamma}^{\alpha}\right\}$.

\subsubsection{Nonholonomic torsions in (pseudo) Riemannian geometry}

Prescribing a $\mathrm{N}$-connection $\mathbf{N}$, we can work equivalently with $\nabla$ and, for instance, the so-called canonical d-connection, $\widehat{\mathbf{D}}$, when

$$
(\mathbf{g}, \mathbf{N}) \rightarrow\left\{\begin{array}{l}
\nabla: \quad \nabla \mathbf{g}=0 ; \nabla \mathcal{T}=0 \\
\widehat{\mathbf{D}}: \quad \widehat{\mathbf{D}} \mathbf{g}=0 ; h \widehat{\mathcal{T}}=0, v \widehat{\mathcal{T}}=0, h v \widehat{\mathcal{T}} \neq 0
\end{array}\right.
$$

and all geometric objects are completely defined by the same metric structure. In above formulas, $h \widehat{\mathcal{T}}$ and $v \widehat{\mathcal{T}}$ are torsion components on, respectively, $\mathrm{h}$ - and v-subspace when a nontrivial $h v \widehat{\mathcal{T}}$ is possible for horizontal-vertical components because of nonholonomic structure. 
The Ricci tensors of $\widehat{\mathbf{D}}$ and $\nabla$ are computed in the standard form, $\widehat{\mathcal{R}} i c=$ $\left\{\widehat{\mathbf{R}}_{\alpha \beta}:=\widehat{\mathbf{R}}_{\alpha \beta \gamma}^{\gamma}\right\}$ and Ric $=\left\{R_{\alpha \beta}:=R_{\alpha \beta \gamma}^{\gamma}\right\}$. The Ricci d-tensor $\widehat{\mathcal{R}} i c$ is characterized by $\mathrm{N}$-adapted coefficients

$$
\widehat{\mathbf{R}}_{\alpha \beta}=\left\{\widehat{R}_{i j}:=\widehat{R}_{i j k}^{k}, \widehat{R}_{i a}:=-\widehat{R}_{i k a}^{k}, \widehat{R}_{a i}:=\widehat{R}_{a i b}^{b}, \widehat{R}_{a b}:=\widehat{R}_{a b c}^{c}\right\},
$$

and scalar curvature,

$$
\widehat{\mathbf{R}}:=\mathbf{g}^{\alpha \beta} \widehat{\mathbf{R}}_{\alpha \beta}=g^{i j} \widehat{R}_{i j}+g^{a b} \widehat{R}_{a b},
$$

which is alternative to the LC-scalar curvature, $R:=\mathbf{g}^{\alpha \beta} R_{\alpha \beta}$.

Any (pseudo) Riemannian geometry can be equivalently described by both geometric data $(\mathbf{g}, \nabla)$ and/or $(\mathbf{g}, \mathbf{N}, \widehat{\mathbf{D}})$ and canonical distortion relations, $\widehat{\mathcal{R}}=\nabla \mathcal{R}+\nabla \mathcal{Z}$ and $\widehat{\mathcal{R}} i c=$ Ric $+\widehat{\mathcal{Z}} i c$. Nevertheless, gravitational and matter field theories for different $\nabla$ and $\widehat{\mathbf{D}}$ are not equivalent if certain additional conditions are not imposed. The Einstein d-tensor of $\widehat{\mathbf{D}}$,

$$
\widehat{\mathbf{E}}_{\alpha \beta}:=\widehat{\mathbf{R}}_{\alpha \beta}-\frac{1}{2} \mathbf{g}_{\alpha \beta} \widehat{\mathbf{R}}
$$

and energy-momentum d-tensors, $\widehat{\mathbf{T}}_{\alpha \beta}:=-\frac{2}{\sqrt{\left|\mathbf{g}_{\mu \nu}\right|}} \frac{\delta\left(\sqrt{\left|\mathbf{g}_{\mu \nu}\right|}{ }^{m} \widehat{\mathcal{L}}\right)}{\delta \mathbf{g}^{\alpha \beta}}$ constructed for a Lagrange density ${ }^{m} \mathcal{L}$, are different from those used for $\nabla$. For a nontrivial $\mathrm{N}$-connection structure, we construct a nonholonomic deformation of the Einstein gravity, with $\nabla \rightarrow \widehat{\mathbf{D}}=\nabla+\widehat{\mathbf{Z}}$, with gravitational field equations

$$
\widehat{\mathbf{R}}_{\alpha \beta}=\kappa^{2}\left(\widehat{\mathbf{T}}_{\alpha \beta}-\frac{1}{2} \mathbf{g}_{\alpha \beta} \widehat{\mathbf{T}}\right),
$$

for a conventional gravitational constant $\kappa^{2}$ and $\widehat{\mathbf{T}}:=\mathbf{g}^{\mu \nu} \widehat{\mathbf{T}}_{\mu \nu}$.

The equations (12) contain nontrivial $\mathrm{N}$-adapted coefficients for the torsion,

$\widehat{T}^{i}{ }_{j k}=\widehat{L}_{j k}^{i}-\widehat{L}_{k j}^{i}, \widehat{T}^{i}{ }_{j a}=\widehat{C}_{j b}^{i}, \widehat{T}^{a}{ }_{j i}=-\Omega^{a}{ }_{j i}, \widehat{T}_{a j}^{c}=\widehat{L}_{a j}^{c}-e_{a}\left(N_{j}^{c}\right), \widehat{T}_{b c}^{a}=\widehat{C}_{b c}^{a}-\widehat{C}_{c b}^{a}$.

which are determined completely by the coefficients $\mathbf{g}_{\alpha \beta}$ (2), $N_{i}^{a}$ and their derivatives. Levi-Civita (LC)-configurations can be extracted from certain classes of solutions of the equations (12) if additional conditions are imposed resulting in zero values for the canonical $\mathrm{d}$-torsion, $\widehat{\mathcal{T}}=0$.

\subsection{Equivalent modelling of MGTs}

Let us state the conditions when the three classes of MGTs can be equivalently modelled by the solutions of certain corresponding field equations. 
We consider such actions and functionals for gravitational, ${ }^{g} S$, and matter, ${ }^{m} S$, fields:

$$
\begin{aligned}
\mathcal{S} & ={ }^{g} \mathcal{S}+{ }^{m} \mathcal{S}=\frac{1}{2 \kappa^{2}} \int f(R) \sqrt{|g|} d^{4} u+\int{ }^{m} \mathcal{L} \sqrt{|g|} d^{4} u \\
& ={ }^{g} \widehat{\mathbf{S}}+{ }^{m} \widehat{\mathbf{S}}=\frac{1}{2 \kappa^{2}} \int \widehat{\mathbf{f}}(\widehat{\mathbf{R}}) \sqrt{|\mathbf{g}|} \mathbf{d}^{4} u-\frac{\check{\mu}^{2}}{4} \mathcal{U}\left(\mathbf{g}_{\mu \nu}, \mathcal{K}_{\alpha \beta}\right)+\int{ }^{m} \widehat{\mathbf{L}} \sqrt{|\mathbf{g}|} \mathbf{d}^{4} u \\
& ={ }^{g} \check{\mathbf{S}}+{ }^{m} \check{\mathbf{S}}=\frac{1}{2 \kappa^{2}} \int \check{\mathbf{R}} \sqrt{|\check{\mathbf{g}}|} \mathbf{d}^{4} u+\check{\Lambda} \int \sqrt{|\check{\mathbf{g}}|} \mathbf{d}^{4} u
\end{aligned}
$$

defined on a pseudo-Riemannian manifold $\mathbf{V}$ by the same metric field $\mathbf{g}=$ $\left\{g_{\mu \nu}\right\}$, but up to different classes of nonholonomic frame transformations and deformations with $(g, \nabla) \sim(\mathbf{g}, \mathbf{N}, \widehat{\mathbf{D}}) \sim(\check{\mathbf{g}}, \check{\mathbf{N}}, \check{\mathbf{D}})$. The equality symbols specify that we shall work with one action written in three different forms when the matter ${ }^{m} \check{\mathbf{S}}$ is encoded into and effective term with $\check{\Lambda}$. We shall explain below the nature of the all terms in above actions.

The theories with actions of type (14) generalize the so-called modified $f(R)$ gravity, see reviews and original results in [9], and the ghostfree massive gravity (by de Rham, Gabadadze and Tolley, dRGT) [19]. We use the natural units of $\hbar=c=1$ and the square of the Planck mass is $M_{P l}^{2}=1 / 8 \pi G=\kappa^{-2}$ with the $4-\mathrm{d}$ Newton constant $G$. It is written $\mathbf{d}^{4} u$ in bold face instead of $d^{4} u$ because the $\mathrm{N}$-elongated differentials are used (44) and $\dot{\mu}=$ const is taken for the mass of the graviton. We can fix conditions of the type

$$
\widehat{\mathbf{f}}(\widehat{\mathbf{R}})-\frac{\stackrel{\circ}{\mu}^{2}}{4} \mathcal{U}\left(\mathbf{g}_{\mu \nu}, \mathcal{K}_{\alpha \beta}\right)=\mathbf{f}(\widehat{\mathbf{R}}), \quad \text { or } \widehat{\mathbf{f}}(\widehat{\mathbf{R}})=f(R), \quad \text { or } \widehat{\mathbf{f}}(\widehat{\mathbf{R}})=R,
$$

which allows us to extract LC-configurations depending on the type of models and classes of solutions we are going to study. To analyze possible scenarios of dynamical supersymmetry breaking it is more convenient to work with geometric variables and field equations determined by $\widehat{\mathbf{D}}$ and $\widehat{\mathbf{R}}$.

The equations of motion for massive MGT (14) can be written in the variables $(\mathbf{g}, \mathbf{N}, \widehat{\mathbf{D}})$,

$$
\left(\partial_{\widehat{\mathbf{R}}} \widehat{\mathbf{f}}\right) \widehat{\mathbf{R}}_{\mu \nu}-\frac{1}{2} \widehat{\mathbf{f}}(\widehat{\mathbf{R}}) \mathbf{g}_{\mu \nu}+\stackrel{\circ}{\mu}^{2} \mathbf{X}_{\mu \nu}=\kappa^{2} \widehat{\mathbf{T}}_{\mu \nu},
$$

where $M_{P l}$ is the Plank mass. For simplicity, we shall consider matter actions which depend only on the coefficients of a metric field and not on its derivatives,

$$
\widehat{\mathbf{T}}^{\alpha \beta}={ }^{m} \widehat{\mathbf{L}} \mathbf{g}^{\alpha \beta}+2 \delta\left({ }^{m} \widehat{\mathbf{L}}\right) / \delta \mathbf{g}_{\alpha \beta},
$$

see details of the variational methods in [19]; we shall follow some conventions from [20]). We are able to construct solutions in MGT in explicit form if we 
fix the coefficients $\left\{N_{i}^{a}\right\}$ of $\mathbf{N}$ and local frames for $\widehat{\mathbf{D}}$ when $\widehat{\mathbf{R}}=$ const and $\partial_{\alpha} \widehat{\mathbf{f}}(\widehat{\mathbf{R}})=\left(\partial_{\widehat{\mathbf{R}}} \widehat{\mathbf{f}}\right) \times \partial_{\alpha} \widehat{\mathbf{R}}=0$ but, in general, $\partial_{\alpha} f(R) \neq 0$. For $\widehat{\mathbf{D}} \rightarrow \nabla$, we obtain $\widehat{\mathbf{R}}_{\mu \nu} \rightarrow R_{\mu \nu}$ with a standard Ricci tensor $R_{\mu \nu}$ for $\nabla$.

The effective energy-momentum tensor $\mathbf{X}_{\mu \nu}$ in (17) is defined by the potential of the graviton $\mathcal{U}=\mathcal{U}_{2}+\alpha_{3} \mathcal{U}_{3}+\alpha_{4} \mathcal{U}_{4}$, where $\alpha_{3}$ and $\alpha_{4}$ are free parameters. The values $\mathcal{U}_{2}, \mathcal{U}_{3}$ and $\mathcal{U}_{4}$ are certain polynomials of traces of some other polynomials of the matrix $\mathcal{K}_{\mu}^{\nu}=\delta_{\mu}^{\nu}-\left(\sqrt{g^{-1} \Sigma}\right)_{\mu}^{\nu}$ for a tensor determined by four Stückelberg fields $\phi \underline{\underline{\mu}}$ as

$$
\Sigma_{\mu \nu}=\left(\mathbf{e}_{\mu} \phi^{\underline{\mu}}\right)\left(\mathbf{e}_{\nu} \phi^{\underline{\nu}}\right) \eta_{\underline{\mu \nu}},
$$

when $\eta_{\underline{\mu} \underline{\underline{\nu}}}=(1,1,1,-1)$. Following a series of arguments presented in [20], when the parameters are chosen $\alpha_{3}=(\alpha-1) / 3, \alpha_{4}=\left(\alpha^{2}-\alpha+1\right) / 12$ and which is useful for avoiding potential ghost instabilities, we can fix

$$
\mathbf{X}_{\mu \nu}=\alpha^{-1} \mathbf{g}_{\mu \nu}
$$

with a constant $\alpha$ which induces an additional term for an (effective) cosmological constant. For such parameterizations, the field equations in MGT can be written in an effective form which is very similar to (12),

$$
\widehat{\mathbf{R}}_{\mu \nu}=\widehat{\mathcal{Y}}_{\alpha \beta}
$$

with effective source

$$
\widehat{\mathcal{Y}}_{\alpha \beta}=\left(\partial_{\widehat{\mathbf{R}}} \widehat{\mathbf{f}}\right)^{-1}\left[\left(\frac{1}{2} \widehat{\mathbf{f}}-\stackrel{\mu}{ }^{2}\right) \mathbf{g}_{\alpha \beta}+\kappa^{2} \widehat{\mathbf{T}}_{\alpha \beta}\right] .
$$

Redefining the generating function and effective source (see below formula (42) and related details) we can encode the MGT contributions into generic off-diagonal terms of an effective d-metric ğ (2) and canonical d-connection $\check{\mathrm{D}}$ as solutions of the nonholonomically deformed Einstein manifolds with field equations determined by the action (15),

$$
\check{\mathbf{R}}_{\mu \nu}=\check{\Lambda}_{\check{g}_{\alpha \beta}} .
$$

In the next subsection, we shall explain how the equations and solutions of (20) can be transformed into similar data as that described by eq-(22) and conversely.

\subsection{The AFDM in MGT}

We summarize the anholonomic frame method, AFDM, which allows us to construct generic off-diagonal solutions in MGTs [11, 6]. 


\subsubsection{Decoupling property for the canonical $\mathrm{d}$-connection with respect to $\mathrm{N}$-adapted frames}

We can consider such matter fields and $\mathrm{N}$-adapted frame and coordinate transformations when a source (21) is parametrized as

$$
\begin{aligned}
\widehat{\mathcal{Y}}_{\alpha \beta} & \rightarrow \operatorname{diag}\left[\Upsilon_{1}=\Upsilon_{2}, \Upsilon_{2}={ }^{h} \Upsilon\left(x^{i}\right), \Upsilon_{3}=\Upsilon_{4}, \Upsilon_{4}={ }^{v} \Upsilon\left(x^{i}, y^{4}\right)\right] \\
\text { or } & \rightarrow \Lambda \mathbf{g}_{\alpha \beta}, \Lambda=\text { const. }
\end{aligned}
$$

For simplicity, we can consider effective matter sources and "prime" metrics with a Killing symmetry in $\partial / \partial_{3}$, i.e. when the effective matter sources and $\mathrm{d}-$ metrics do not depend on the coordinate $y^{3} 6$ In brief, the partial derivatives $\partial_{\alpha}=\partial / \partial u^{\alpha}$ on a $4-$ d manifold will be written $s^{\bullet}=\partial s / \partial x^{1}, s^{\prime}=$ $\partial s / \partial x^{2}, s^{*}=\partial s / \partial y^{3}, s^{\diamond}=\partial s / \partial y^{4}$.

The nontrivial components of the Ricci d-tensor (9) for a d-metric ansatz (2) with coefficients

$$
\mathbf{g}_{i j}=\operatorname{diag}\left[g_{i}=\epsilon_{i} e^{\psi\left(x^{k}\right)}\right], g_{a b}=\operatorname{diag}\left[\omega^{2}\left(u^{\alpha}\right) h_{a}\left(x^{k}, y^{4}\right)\right]
$$

$\epsilon_{i}= \pm 1$ depending on the signature, with

$$
\mathbf{e}_{i} \omega=\partial_{i} \omega-n_{i} \omega^{*}-w_{i} \omega^{\diamond}=0,
$$

for $N_{i}^{3}=n_{i}\left(x^{k}\right)$ and $N_{i}^{4}=w_{i}\left(x^{k}, y^{4}\right)$, are

$$
\begin{aligned}
\widehat{R}_{1}^{1} & =\widehat{R}_{2}^{2}=\frac{1}{2 g_{1} g_{2}}\left[-g_{2}^{\bullet \bullet}+\frac{g_{1}^{\bullet} g_{2}^{\bullet}}{2 g_{1}}+\frac{\left(g_{2}^{\bullet}\right)^{2}}{2 g_{2}}-g_{1}^{\prime \prime}+\frac{g_{1}^{\prime} g_{2}^{\prime}}{2 g_{2}}+\frac{\left(g_{1}^{\prime}\right)^{2}}{2 g_{1}}\right], \\
\widehat{R}_{3}^{3} & =\widehat{R}_{4}^{4}=\frac{1}{2 h_{3} h_{4}}\left[-h_{3}^{\diamond}+\frac{\left(h_{3}^{\diamond}\right)^{2}}{2 h_{3}}+\frac{h_{3}^{\diamond} h_{4}^{\diamond}}{2 h_{4}}\right], \widehat{R}_{3 k}=\frac{h_{3}}{2 h_{4}} n_{k}^{\diamond \diamond}+\left(\frac{h_{3}}{h_{4}} h_{4}^{\diamond}-\frac{3}{2} h_{3}^{\diamond}\right) \frac{n_{k}^{\diamond}}{2 h_{4}}, \\
\widehat{R}_{4 k} & =\frac{w_{k}}{2 h_{3}}\left[h_{3}^{\diamond}-\frac{\left(h_{3}^{\diamond}\right)^{2}}{2 h_{3}}-\frac{h_{3}^{\diamond} h_{4}^{\diamond}}{2 h_{4}}\right]+\frac{h_{3}^{\diamond}}{4 h_{3}}\left(\frac{\partial_{k} h_{3}}{h_{3}}+\frac{\partial_{k} h_{4}}{h_{4}}\right)-\frac{\partial_{k} h_{3}^{\diamond}}{2 h_{3}} .
\end{aligned}
$$

The d-torsion (13) vanishes if the Levi-Civita (LC) conditions are satisfied : $\widehat{L}_{a j}^{c}=e_{a}\left(N_{j}^{c}\right), \widehat{C}_{j b}^{i}=0, \Omega^{a}{ }_{j i}=0$. Given the ansatz in (25), we can write these equations in the form

$$
\begin{aligned}
w_{i}^{\diamond} & =\left(\partial_{i}-w_{i} \partial_{4}\right) \ln \sqrt{\left|h_{4}\right|},\left(\partial_{i}-w_{i} \partial_{4}\right) \ln \sqrt{\left|h_{3}\right|}=0 \\
\partial_{k} w_{i} & =\partial_{i} w_{k}, n_{i}^{\diamond}=0, \partial_{i} n_{k}=\partial_{k} n_{i} .
\end{aligned}
$$

\footnotetext{
${ }^{6}$ Solutions with dependencies on $y^{3}$ and non-Killing configurations are studied in [6].
} 
Using the parametrizations (23) $-(26)$ for $h_{a}^{\diamond} \neq 0$ and ${ }^{h} \Upsilon,{ }^{v} \Upsilon \neq 0$, the MGT fied equations (20) can be written in the form

$$
\begin{aligned}
\epsilon_{1} \psi^{\bullet \bullet}+\epsilon_{2} \psi^{\prime \prime} & =2^{h} \Upsilon \\
\phi^{\diamond} h_{3}^{\diamond} & =2 h_{3} h_{4}{ }^{v} \Upsilon \\
n_{i}^{\diamond}+\gamma n_{i}^{\diamond} & =0, \\
\beta w_{i}-\alpha_{i} & =0, \\
\partial_{i} \omega-\left(\partial_{i} \phi / \phi^{\diamond}\right) \omega^{\diamond} & =0 .
\end{aligned}
$$

In (31) and (32) the coefficients are defined as

$$
\alpha_{i}=h_{3}^{\diamond} \partial_{i} \phi, \beta=h_{3}^{\diamond} \phi^{\diamond}, \gamma=\left(\ln \left|h_{3}\right|^{3 / 2} /\left|h_{4}\right|\right)^{\diamond},
$$

where

$$
\phi=\ln \mid h_{3}^{\diamond} / \sqrt{\left|h_{3} h_{4}\right|}, \text { and } / \text { or } \Phi:=e^{\phi},
$$

can be considered as a generating function (see below).

There is a decoupling property (with respect to the $\mathrm{N}$-adapted frames and for the canonical $d$-connection) of the above system of nonlinear PDEs. This can be exploited in this form: 1) integrating the 2-d Laplace, or d'Alambert, equation (29) we find solutions for the $h$-coefficients of the d-metric, $g_{i}=$ $\epsilon_{i} e^{\psi\left(x^{k}\right)}$, and 2) the solutions for the $v$-coefficients of the d-metric, $h_{a}$, can be found from (30) and (35). 3) Then the $\mathrm{N}$-connection coefficients $w_{i}$ and $n_{i}$ can be computed respectively from (31) and (32).

\subsubsection{Generating off-diagonal solutions}

Let us explain how the equations (29) $-(33)$ can be solved, respectively for any sources ${ }^{h} \Upsilon\left(x^{k}\right)$ and ${ }^{h} \Upsilon\left(x^{i}\right)$ and ${ }^{v} \Upsilon\left(x^{i}, y^{4}\right)$ and generating function $\phi\left(x^{k}, y^{4}\right)$ : The 2-d Laplace/ d'Alambert equation (29) is well-known for physicists and can be solved for any ${ }^{h} \Upsilon\left(x^{k}\right)$.

We re-write the system (30) and (35) in the form

$$
h_{3} h_{4}=\phi^{\diamond} h_{3}^{\diamond} / 2^{v} \Upsilon \text { and }\left|h_{3} h_{4}\right|=\left(h_{3}^{\diamond}\right)^{2} e^{-2 \phi} .
$$

Inserting the first equation into the second one, we can express $\left|h_{3}^{\diamond}\right|=\frac{\left(e^{2 \phi}\right)^{\diamond}}{\left.4\right|^{v} \Upsilon \mid}=$ $\frac{\Phi^{\triangleright} \Phi}{2\left|{ }^{v} \Upsilon\right|}$. This can be integrated with respect to $y^{4}$ which results in

$$
h_{3}={ }^{0} h_{3}+\frac{\epsilon_{3} \epsilon_{4}}{4} \int d y^{4} \frac{\left(\Phi^{2}\right)^{\diamond}}{v \Upsilon}
$$

where ${ }^{0} h_{3}={ }^{0} h_{3}\left(x^{k}\right)$ and $\epsilon_{3}, \epsilon_{4}= \pm 1$. Using again the first equation from (36), we obtain

$$
h_{4}=\frac{1}{2^{v} \Upsilon} \frac{\Phi^{\diamond}}{\Phi} \frac{h_{3}^{\diamond}}{h_{3}}
$$


Having the solutions (37) and (38) for $h_{a}[\Phi]$, we can solve equation (31) by integrating twice with respect to $d y^{3}$ and express

$$
n_{k}={ }_{1} n_{k}+{ }_{2} n_{k} \int d y^{4} h_{4} /\left(\sqrt{\left|h_{3}\right|}\right)^{3},
$$

where ${ }_{1} n_{k}\left(x^{i}\right),{ }_{2} n_{k}\left(x^{i}\right)$ are integration functions. The rest of the $\mathrm{N}$-connection coefficients can be found from equation (32) with the coefficients in (34). The solutions of such algebraic linear equations can be expressed as

$$
w_{i}=\frac{\partial_{i} \phi}{\phi^{\diamond}}=\frac{\partial_{i} \Phi}{\Phi^{\diamond}}=\frac{\partial_{i}\left(\Phi^{2}\right)}{\left(\Phi^{2}\right)^{\diamond}} .
$$

In general form, the metrics defining the off-diagonal solutions of the MGTs equations (20) with $\omega$ subjected to the condition (26) are parametrized as

$$
\begin{aligned}
d s^{2}= & \epsilon_{i} e^{\psi\left[{ }^{h} \Upsilon\right]}\left(d x^{i}\right)^{2}+\omega^{2}\left(u^{\beta}\right) h_{3}\left[\Phi,{ }^{v} \Upsilon\right]\left(d y^{3}+n_{k}\left[\Phi,{ }^{v} \Upsilon\right] d x^{k}\right)^{2} \\
& +\omega^{2}\left(u^{\beta}\right) h_{4}\left[\Phi,{ }^{v} \Upsilon\right]\left(d y^{4}+w_{k}[\Phi] d x^{k}\right)^{2},
\end{aligned}
$$

with the functional form of the coefficients depending on the generating functions $\omega$ and $\Phi$, the sources ${ }^{h} \Upsilon$ and ${ }^{v} \Upsilon$ and the integration functions and parameters as computed above. Such metrics cannot be diagonalized by coordinate transformations if the anholonomy coefficients $W_{\alpha \beta}^{\gamma}$ (5) are not trivial. For (41), the nonholonomically induced torsion (13) is not zero. If we fix ${ }_{2} n_{k}=0$, we are able to find $n_{k}={ }_{1} n_{k}\left(x^{i}\right)$ which have zero torsion limits if the coefficients of the $\mathrm{d}$-metric are chosen to satisfy, additionally, the conditions (28), see Refs. [11, 6] for the explicit geometric methods and examples.

\subsubsection{Encoding MGTs as nonholonomic Einstein manifolds}

There is an important property of the system (36) and solutions (41). We can always fix a nontrivial constant $\check{\Lambda}$ and re-define the generating function, $\Phi \longleftrightarrow \check{\Phi}$, using the formulas

$$
\begin{aligned}
\check{\Phi}^{2} & =\check{\Lambda}^{-1}\left[\Phi^{2}\left|{ }^{v} \Upsilon\right|+\left.\left.\int d y^{4} \Phi^{2}\right|^{v} \Upsilon\right|^{\diamond}\right], \\
\Phi^{2} & =\left.\left.|\check{\Lambda}|{ }^{v} \Upsilon\right|^{-2} \int d y^{4} \check{\Phi}^{2}\right|^{v} \Upsilon \mid,
\end{aligned}
$$

when $\left(\Phi^{2}\right)^{\diamond} /{ }^{v} \Upsilon \mid=\left(\check{\Phi}^{2}\right)^{\diamond} / \check{\Lambda}$. We can simplify the formulas for $h_{a} \rightarrow \check{h}_{a}$, see (37) and (38), including ${ }^{0} h_{3}\left(x^{k}\right)$ in $\check{\Phi}$ and parametrizing

$$
\check{h}_{3}[\check{\Phi}]=\frac{\check{\Phi}^{2}}{4 \check{\Lambda}} \text { and } \check{h}_{4}[\check{\Phi}]=\frac{\left(\check{\Phi}^{\diamond}\right)^{2}}{\check{\Lambda} \Phi^{2}}=\frac{\left|\check{\Phi}^{\diamond} v \Upsilon\right|^{2}}{\check{\Lambda}|\check{\Lambda}| \int d y^{4} \check{\Phi}^{2}\left|{ }^{v} \Upsilon\right|} \text {. }
$$


The conformal factor $\psi\left[{ }^{h} \Upsilon\right]$ with source ${ }^{h} \Upsilon$ is reparametrized by the $2-$ d coordinate transformations $x^{i} \rightarrow \check{x}^{i}\left(x^{k}\right)$ into $\check{\psi}[\check{\Lambda}]$, when equation (29) transforms into

$$
\epsilon_{1} \check{\psi}^{\bullet \bullet}+\epsilon_{2} \check{\psi}^{\prime \prime}=2 \check{\Lambda}
$$

where, for instance, $\check{\psi} \bullet=\partial \check{\psi} / \partial \check{x}^{1}$ and $\check{\psi}^{\prime}=\partial \check{\psi} / \partial \check{x}^{2}$.

The N-connection coefficients $n_{i} \rightarrow \check{n}_{i}$ and $w_{i} \rightarrow \check{w}_{i}$ can be computed as functionals of $\check{\Phi}$ and the data $\left(\check{\Lambda},{ }^{v} \Upsilon\right)$ by substituting respectively $\check{h}_{a}$ (44) and $\Phi^{2}$ (43) into formulas (39) and (40). The equations (26) for the re-defined conformal factor $\omega \rightarrow \check{\omega}$ transform into

$$
\check{\mathbf{e}}_{i} \check{\omega}=\partial_{i} \check{\omega}-\check{n}_{i} \check{\omega}^{*}-\check{w}_{i} \check{\omega}^{\diamond}=0 .
$$

We obtain a class of $d-$ metrics

$$
\begin{aligned}
d s^{2}= & \epsilon_{i} e^{\check{\psi}[\check{\Lambda}]}\left(d \check{x}^{i}\right)^{2}+\check{\check{\omega}}^{2}\left(\check{x}^{i}, y^{a}\right) \check{h}_{3}[\check{\Phi}, \check{\Lambda}]\left(d y^{3}+\check{n}_{k}\left[\check{\Phi}, \check{\Lambda},{ }^{v} \Upsilon\right] d \check{x}^{k}\right)^{2} \\
& +\check{\omega}^{2}\left(\check{x}^{i}, y^{a}\right) \check{h}_{4}\left[\check{\Phi}, \check{\Lambda},{ }^{v} \Upsilon\right]\left(d y^{4}+\check{w}_{k}\left[\check{\Phi}, \check{\Lambda},{ }^{v} \Upsilon\right] d \check{x}^{k}\right)^{2}
\end{aligned}
$$

defining effective (nonholonomic) Einstein spaces (22) with $\check{\mathbf{R}}_{\beta}^{\alpha}$ computed with the canonical d-connection $\check{\text { D. }}$.

The metrics (46) encode contributions from $f$-modified and/or massive gravity via ${ }^{v} \Upsilon$ (23). Using re-definitions of the generating functions and (effective) sources of type (42) and (43), we construct nonholonomic deformations of such generic off-diagonal metrics into the respective classes of solutions described by eq-(41) or the MGT field equations (20).

\subsubsection{Examples: off-diagonal solutions and torsion}

We analyzed three different classes of off-diagonal solutions (46) encoding MGT configurations characterized by different symmetries with possible physical implications in classical gravity and dynamical breaking of supergravity. We proved in [24] that (for certain general conditions) such solutions can be encoded as solitonic hierarchies and provided various applications in black hole physics, string and brane gravity with wormholes, flux tubes, nonlinear cosmological solutions etc. In order to study nonlinear effects, we shall consider three and two dimensional (3-d and 2-d) solitonic waves with nontrivial nonholonomically induced torsion of type (13). If the zero torsion conditions (28) are imposed, we get LC-configurations which may result in dynamical breaking of local supersymmetry only if additional sources for the torsion fields are introduced.

Let us parameterize $u^{\alpha}=\left(x^{i}, y^{3}, y^{4}=t\right)$ for a time-like coordinate $t$. There are two well known examples when a function $q\left(x^{i}, t\right)$ describes solutions of three-dimensional $(3-\mathrm{d})$ solitonic waves in terms of solutions of 
- the Kadomtsev-Petviashvili, KdP, equation [25]

$$
q^{\diamond \diamond} \pm\left(q^{\bullet}+6 q q^{\prime}+q^{\prime \prime \prime}\right)^{\prime}=0
$$

- the $2+1$ sine-Gordon equation,

$$
q^{\diamond}+q^{\prime \prime}+q^{\bullet \bullet}=\sin (q)
$$

or other type of solitonic waves and hierarchies which Killing symmetries in $y^{3}$, or with non-Killing symmetries in the presence of a nontrivial factor $\check{\omega}^{2}\left(x^{i}, y^{a}\right)$.

Generating functions for solitonic waves (for arbitrary sources from MGTs): This class of solutions is defined by $\check{h}=\check{\Phi}^{2} / 4 \check{\Lambda}=q$ in (44). Considering, for simplicity, $\check{\omega}=1$ and substituting $\check{\Phi}=2 \sqrt{|\check{\Lambda} q|}$, we generate a subclass of (46) of solitonic metrics with Killing symmetry in $y^{3}$

$$
\begin{aligned}
d s^{2} & =\epsilon_{i} e^{\check{\psi}[\check{\Lambda}]}\left(d \check{x}^{i}\right)^{2}+q\left(d y^{3}+\check{n}_{k} d \check{x}^{k}\right)^{2}+\frac{\left(q^{\diamond}\right)^{2}}{4 \check{\Lambda}|\check{\Lambda}| q} \frac{\left({ }^{v} \Upsilon\right)^{2}}{\int\left|q^{v} \Upsilon\right| d t}\left(d t+\frac{\partial_{k}|q|}{|q|^{\diamond}} d \check{x}^{k}\right)^{2} \\
\check{n}_{k} & ={ }_{1} n_{k}\left(\check{x}^{i}\right)+{ }_{2} n_{k}\left(\check{x}^{i}\right) \int d t \frac{\left(q^{\diamond}\right)^{2}}{|q|^{5 / 2}}\left(\int_{0}^{t} d t^{\prime}\left|q^{v} \Upsilon\left(\check{x}^{i}, t^{\prime}\right)\right|\right)^{-1}
\end{aligned}
$$

where the integration functions ${ }_{2} n_{k}\left(\check{x}^{i}\right)$ were re-defined to include all constants from $h_{4} /\left(\sqrt{\left|h_{3}\right|}\right)^{3}$. If ${ }_{2} n_{k}\left(\check{x}^{i}\right) \neq 0$, it is not possible to find nonholonomic distributions resulting in LC-configurations. We need to impose additional constraints on ${ }_{1} n_{k}\left(\check{x}^{i}\right)$ and source ${ }^{v} \Upsilon$ in order to get nonholonomically induced zero torsion configurations. Such special cases may not result in dynamical supersymmetry breaking.

The solitonic metrics (49) define solitonic solutions with Killing symmetry in $y^{3}$ for any source ${ }^{v} \Upsilon\left(\check{x}^{i}, t\right)$ containing information of MGTs. The value $\check{\Lambda}$ is an effective cosmological constant which can be fixed in order to characterize the "intensity" of nonlinear interactions. It contributes directly to the bosonic effective potential (see Section 4).

Vertical conformal non-Killing deformations of $3-\mathrm{d}$ solitonic metrics: We can introduce a conformal $v$-factor $\check{\omega}\left(\check{x}^{i}, y^{3}, t\right)$ which is a solution of the system of first order PDEs (45),

$$
\begin{aligned}
\check{\omega}^{\bullet}-\check{n}_{1} \check{\omega}^{*}-|q|^{\bullet} \check{\omega}^{\diamond} /|q|^{\diamond}=0, \\
\check{\omega}^{\prime}-\check{n}_{2} \check{\omega}^{*}-|q|^{\prime} \check{\omega}^{\diamond} /|q|^{\diamond}=0,
\end{aligned}
$$


where $\check{n}_{k}$ can be taken as in (49). It can be solved for $\check{\omega}$ for very general classes of solitonic and integrations functions contained in $q$ and $\check{n}_{k}$. Conventionally, such metrics can be parameterized in the form

$$
\begin{aligned}
d s^{2}= & \epsilon_{i} e^{\check{\psi}[\check{\Lambda}]}\left(d \check{x}^{i}\right)^{2}+\check{\omega}^{2}\left(u^{\alpha}\right) q\left(d y^{3}+\check{n}_{k}\left[q, x^{k},{ }^{v} \Upsilon\right] d \check{x}^{k}\right)^{2} \\
& +\check{\omega}^{2}\left(u^{\alpha}\right) \frac{\left(q^{\diamond}\right)^{2}}{4 \check{\Lambda}|\check{\Lambda}| q} \frac{\left({ }^{v} \Upsilon\right)^{2}}{\int\left|q^{v} \Upsilon\right| d t}\left(d t+\frac{\partial_{k}|q|}{|q|^{\diamond}} d \check{x}^{k}\right) .
\end{aligned}
$$

A nontrivial factor $\breve{\omega}$ polarizes the solutions of the nonholonomic Einstein equations with effective cosmological constant $\check{\Lambda}$. It contains contributions of MGTs sources ${ }^{v} \Upsilon$ encoded in a nonlinear wave form in the diagonal and off-diagonal components of the metrics and with nonholonomically induced torsion.

Off-diagonal "conditional" solitonic configurations: Both classes of metrics (49) and (51) can be constructed for arbitrary classes of nonlinear (solitonic) waves which do not require the condition that the gravitational field equations in a MGT are transformed, for instance, into a solitonic equation of type (48), or (47). Nevertheless, for certain classes of nonhololonomic distributions the generalized Einstein equations can be reduced to certain effective 3-d solitonic equations.

Let us consider the equations (40) in the form $\Phi^{\diamond} w_{i}=\partial_{i} \Phi$. We chose a generating function

$$
\Phi=Q\left(x^{2}, t\right) s\left(x^{2}, t\right),
$$

where $Q=\int d x^{2}\left(\widetilde{q}^{\diamond}+\left[\widetilde{q}^{\prime \prime}-\sin (\widetilde{q})\right]\right)$, for $\widetilde{q}\left(x^{2}, t\right)$. This defines a $2-\mathrm{d}$ sine Gordon solution generating off-diagonal Einstein metrics. We get $w_{1}=0$ for $\Phi^{\bullet}=0$ and

$$
\left[Q^{\diamond} s+Q s^{\diamond}\right] w_{2}=s\left(\widetilde{q}^{\diamond \diamond}+\left[\widetilde{q}^{\prime \prime}-\sin (\widetilde{q})\right]\right)+Q s^{\prime} .
$$

If the equation (48) is satisfied, $Q=0$ and $w_{2}\left(x^{2}, t\right)$ is any function, which allows us to generate vacuum off-diagonal solutions, for $\check{\Lambda}=0$, with two Killing vectors $\partial_{1}$ and $\partial_{3}$. Such metrics cannot be represented in a form similar to (51). Nevertheless, the nonholonomic induced torsion (13) may not be zero for such configurations if we do not consider the additional conditions (28) leading to zero torsion. This is an example when the vacuum Einstein metrics may not break the symmetry.

Off-diagonal "conditional" solitonic configurations with nontrivial $\check{\omega}$ : Let us consider a generating function

$$
\phi^{\diamond}=(\ln |\Phi|)^{\diamond}=-2^{v} \Upsilon\left(q^{\prime \prime}+q^{\bullet \bullet}-\sin (q)\right)\left[\left(\ln \left|h_{3}\right|\right)^{\diamond}\right]^{-1}
$$


for arbitrary value $h_{3}^{\diamond} \neq 0$ and nonzero ${ }^{v} \Upsilon$ and $h_{4}=q^{\diamond}$. For such configurations, metrics of type (41) with $\omega=1$ are solutions of the MGT field equations (20) if $q\left(x^{i}, t\right)$ is a solution of 3 -d sine Gordon equations(48).

\section{A $\mathcal{N}=1, d=4$ Modified Supergravity Theory}

Possible nonlinear modifications of gravity are modeled by a nonholonomic background space $\mathbf{V}$, with $\mathrm{N}$-adapted frames $\mathbf{e}_{\mu}, \mathrm{d}$-metric, $\mathbf{g}$, and canonical d-connection, $\widehat{\mathbf{D}}$, structures. These structures can be re-defined equivalently in terms of data of effective Einstein manifolds, $\left(\check{\mathbf{e}}_{\mu}, \check{\mathbf{g}}, \check{\mathbf{D}}\right)$, described by solutions of (22) . In a simple form, a modified supergravity theory/ model, MSGT, can be constructed on such nonholonomic manifolds generalizing the constructions due to Volkov and Akulov [21].

\subsection{Nonholonomic super-Higgs effects and goldstino cou- pling}

The Goldstino field $\lambda$ is a four-component Majorana spin - $1 / 2$ spinor. Using $\mathrm{N}$-adapted operators $\mathbf{e}_{\mu}$, the nonholonomic nonlinear action for the Majorana Goldstino $\lambda$, is postulated as

$$
{ }^{\lambda} \mathcal{L}=-f^{2} \operatorname{det}\left|\delta_{\beta}^{\alpha}+\frac{i}{2 f^{2}} \bar{\lambda} \gamma^{\mu} \mathbf{e}_{\mu} \lambda\right|=-f^{2}-\frac{i}{2} \bar{\lambda} \gamma^{\mu} \mathbf{e}_{\mu} \lambda+\ldots,
$$

when the determinant is expressed via the weak expansion terms. This defines a nonholonomic model of $\mathcal{N}=1$ supergravity off-diagonally coupled to $\lambda$. Such a Lagrangian arises from a spontaneous/ dynamical breaking of global supersymmetry when additional nonholonomic constraints are imposed and the interactions contain certain off-diagonal terms encoding a MGT model. The gamma matrices $\gamma^{\mu}$ in the $\mathrm{N}$-adapted frames are related to the so-called nonholonomic spinor structures and Dirac operators, see details on nonholonomic supermanifolds and d-spinor geometry and applications in MGTs in Ref. 22. In this work, we consider an arbitrary value of the parameter $f=\langle F\rangle$ when the breaking type of the global supersymmetry belongs to the so-called $F$-type with the $F$-term of some chiral superfield acquiring such an expectation value.

The Lagrangian (54) is characterized by a nonlinear nonholonomic realization of global supersymetry with an infinitesimal complex parameter $\alpha$ (and its complex conjugate $\bar{\alpha}$ ), when $\delta \lambda=f \alpha+i \bar{\alpha} f^{-1} \lambda \gamma^{\mu} \mathbf{e}_{\mu} \lambda$. It is possible to generate a mass for the gravitino $\psi_{\alpha}$ through the absorbtion of the Goldstino via a super-Higgs effect. We can introduce local supersymmetry 
by allowing the parameter $\alpha \rightarrow \alpha(u)$ to depend on the spacetime coordinates $u=\left\{u^{\alpha}\right\}$. The equation (54) transforms into

$$
{ }^{\lambda} \mathcal{L}=-f^{2}|\mathbf{e}|-\frac{i}{2} \bar{\lambda} \gamma^{\mu} \mathbf{e}_{\mu} \lambda-\frac{i}{2} \bar{\lambda} \gamma^{\mu} \psi_{\mu}+\ldots,
$$

where $|\mathbf{e}|=\operatorname{det} e^{\underline{\alpha}}{ }_{\alpha}$ denotes the determinant of the vierbein $e^{\frac{\alpha}{\alpha}}$ field which in the $\mathrm{N}$-adapted form defines a decomposition of a d-metric (2), when $\mathbf{g}_{\alpha \beta}=$ $\mathbf{e}^{\underline{\alpha}}{ }_{\alpha} \mathbf{e}^{\underline{\beta}} \eta_{\underline{\alpha} \beta}$ with $\eta_{\underline{\alpha} \underline{\beta}}$ being the Minkowski metric. This action is invariant under following $\mathrm{N}$-adapted supergravity transformations

$$
\delta \lambda=f \alpha(u)+\ldots, \delta e^{\underline{\alpha}}{ }_{\alpha}=-i \kappa \bar{\alpha}(u) \gamma^{\underline{\alpha}} \psi_{\alpha}, \delta \psi_{\alpha}=-2 \kappa^{-1} \mathbf{e}_{\mu} \alpha(u)+\ldots .
$$

We can impose in the $\mathrm{N}$-adapted form the gauge condition $\gamma^{\mu} \psi_{\mu}=0$, when the Goldstino can be gauged away by a suitable redefinition (even in the presence of off-diagonal interactions in MGT) by considering a suitable redefinition of the gravitino field and the vierbein fields. Nevertheless, the term $-f^{2}|\mathbf{e}|$ results in a negative cosmological constant.

\subsection{An Einstein-Cartan like formulation of MSGT}

We have nontrivial torsion fields which can be included in SG theories in a form similar to that for the Einstein-Cartan theory. Nevertheless, we do not need additional field equations for such torsion fields because they are nonholonomically induced by the off-diagonal deformations of the metrics and the respective vielbein fields.

\subsubsection{The nonholonomic supersymmetric Lagrangian}

Let us denote by $\widehat{\omega}_{\mu}^{\underline{\alpha}-\underline{\beta}}$ the canonical spin $\mathrm{d}$-connection determined by $\widehat{\mathbf{D}}$, see details [22] (the constructions are similar to those for $\omega_{\mu}^{\underline{\alpha}}$ - constructed for $\nabla)$. The so-called 1.5 order formalism of the Einstein-Cartan formulation of supergravity [3] is extended to nonholonomic (super) manifolds in the form

$$
{ }^{s g} \mathcal{L}=-\frac{|\mathbf{e}|}{2 \kappa^{2}} \overrightarrow{\mathbf{R}}-\frac{1}{2} \epsilon^{\mu \nu \alpha \beta} \bar{\psi}_{\mu} \gamma_{5} \gamma_{\nu} \widehat{\mathcal{D}}_{\alpha} \psi_{\beta}+\frac{|\mathbf{e}|}{3}\left(\mathbf{A}_{\alpha} \mathbf{A}^{\alpha}-S^{2}-P^{2}\right),
$$

for $\vec{\omega}_{\mu}^{\underline{\alpha} \underline{\beta}}:=\widehat{\omega}_{\mu}^{\underline{\alpha}}-\underline{\beta}\left(\mathbf{e}_{\beta}\right)+\frac{\kappa^{2}}{4}\left(\bar{\psi}_{\mu} \gamma^{\underline{\alpha}} \psi \underline{\underline{\beta}}-\bar{\psi}_{\mu} \gamma^{\underline{\beta}} \psi^{\underline{\alpha}}+\bar{\psi}^{\underline{\alpha}} \gamma_{\mu} \psi \underline{\beta}\right)$ and the covariant spin d-operator $\widehat{\mathcal{D}}_{\alpha}:=\mathbf{e}_{\alpha}+\frac{1}{2} \widehat{\omega}_{\mu}^{\underline{\alpha}}-\left(\mathbf{e}_{\beta}\right) \sigma_{\underline{\alpha}} \underline{\beta}$ defining the canonical d-spinor curvature

$$
\overrightarrow{\mathbf{R}}^{\underline{\alpha \underline{\beta}}}{ }_{\mu \nu}:=\mathbf{e}_{\mu} \vec{\omega}_{\nu}^{\underline{\alpha \underline{\beta}}}-\mathbf{e}_{\nu} \vec{\omega}_{\mu}^{\underline{\alpha} \underline{\beta}}+\vec{\omega}_{\mu}^{\underline{\alpha \tau}} \vec{\omega}_{\nu \underline{\underline{\tau}}}^{\underline{\beta}}-\vec{\omega}_{\nu} \underline{\alpha \tau}^{\vec{\omega}_{\mu \tau}}{ }^{\underline{\beta}}+W_{\mu \nu}^{\gamma} \vec{\omega}_{\gamma}^{\underline{\alpha}}
$$


where $W_{\mu \nu}^{\gamma}$ are the anholonomy coefficients in (5). This allows us to compute the canonical scalar curvature

$$
\overrightarrow{\mathbf{R}}=\mathbf{e}_{\underline{\alpha}}^{\mu} \mathbf{e}_{\underline{\beta}}^{\nu} \overrightarrow{\mathbf{R}}^{\underline{\alpha} \underline{\beta}}{ }_{\mu \nu}=\widehat{\mathbf{R}}\left(\mathbf{e}_{\beta}\right)+\frac{11}{4} \kappa^{4}\left(\bar{\psi}_{\mu} \Gamma^{\mu \nu} \psi_{\nu}\right)^{2}+\ldots,
$$

where $\Gamma^{\mu \nu}=\gamma^{[\mu} \gamma^{\nu]} / 4$ and the fields $\left(\mathbf{A}_{\alpha}, S, P\right)$ comprise a minimal set of auxiliary fields which can be used for the closure of the (super) algebra. The dots ... indicate (as in the original Volkov-Akulov theory) the interaction terms between the gravitino and (modified gravity fields and possible massive) graviton fields and the four-gravitino interactions involving $\gamma_{5}$. The SG action (56) posses a local invariance under $\mathrm{N}$-adapted transforms and a large class of MGTs,

$$
\begin{aligned}
\delta \mathbf{e}^{\frac{\alpha}{\alpha}} & =\frac{\kappa}{2} \bar{\epsilon} \gamma^{\underline{\alpha}} \psi_{\alpha}, \delta \psi_{\nu}=\left(\kappa^{-1} \widehat{\mathcal{D}}_{\nu}+\frac{i}{2} \mathbf{A}_{\nu} \gamma^{5}-\frac{1}{2} \psi_{\nu} \eta\right) \epsilon \\
\delta S & =\frac{1}{4} \bar{\epsilon} \gamma_{\alpha} \widehat{\mathcal{R}}^{\alpha}, \delta P=-\frac{1}{4} \bar{\epsilon} \gamma_{5} \gamma_{\alpha} \widehat{\mathcal{R}}^{\alpha}, \delta \mathbf{A}_{\nu}=\frac{3 i}{4} \bar{\epsilon} \gamma_{5}\left(\widehat{\mathcal{R}}_{\nu}-\gamma_{\nu} \gamma_{\alpha} \widehat{\mathcal{R}}^{\alpha}\right)
\end{aligned}
$$

where $\epsilon$ is a complex parameter, $\eta=\frac{1}{3}\left(-S+i \gamma_{5} P+i \gamma^{\alpha} \mathbf{A}_{\alpha} \gamma_{5}\right)$ and the 'supercovariantised' and nonholonomically deformed gravitino field equation is

$$
\widehat{\mathcal{R}}^{\alpha}=\epsilon^{\alpha \beta \mu \nu} \gamma_{5} \gamma_{\beta}\left(\widehat{\mathcal{D}}_{\mu} \psi_{\nu}-\frac{i}{2} \mathbf{A}_{\nu} \gamma_{5} \psi_{\mu}+\frac{1}{2} \gamma_{\alpha} \eta \psi_{\mu}\right) .
$$

Explicit deformations with respect to the LC-connection can be computed for distortions of the type in (17) when $\widehat{\mathbf{D}}=\nabla+\widehat{\mathbf{Z}}$.

\subsubsection{Off-diagonal mass generation for the gravitino}

The dynamical mass generation for the gravitino can be investigated for an auxiliary scalar field $\sigma$ and its Euler-Lagrange equation and linearizing the four-gravitino interactions in (57),

$$
\frac{|\mathbf{e}|}{2 \kappa^{2}} \overrightarrow{\mathbf{R}} \sim|\mathbf{e}|\left[\frac{1}{2 \kappa^{2}} \widehat{\mathbf{R}}\left(\mathbf{e}_{\beta}\right)-\sigma^{2}-\sqrt{\frac{11}{2}} \frac{\kappa \sigma}{2}\left(\bar{\psi}_{\mu} \Gamma^{\mu \nu} \psi_{\nu}\right)^{2}+\ldots\right] .
$$

We obtain an effective Lagrangian with a nonholonomically Goldstino-induced negative constant (see (155))

$$
\begin{aligned}
{ }^{e f f} \mathcal{L}= & -\frac{|\mathbf{e}|}{2 \kappa^{2}}\left[\widehat{\mathbf{R}}\left(\mathbf{e}_{\beta}\right)+2 \kappa^{2}\left(f^{2}-\sigma^{2}\right)\right] \\
& -\frac{1}{2} \epsilon^{\mu \nu \alpha \beta} \bar{\psi}_{\mu} \gamma_{5} \gamma_{\nu} \widehat{\mathcal{D}}_{\alpha} \psi_{\beta}+\sqrt{\frac{11}{2}} \frac{\kappa \sigma|\mathbf{e}|}{2}\left(\bar{\psi}_{\mu} \psi^{\mu}\right)^{2}+\ldots
\end{aligned}
$$


with distortions in $\widehat{\mathbf{R}}$ and $\widehat{\mathcal{D}}$ which for diagonal configurations in (effective) GR transform into similar values determined by $\nabla$. Such models with dynamical supersymmetry breaking in LC-variables were studied in Refs. [16, 17]. Considering that $\sigma$ may acquire a non-zero vacuum expectation value (vev) $\sigma_{c}:=<{ }^{0} \sigma>+<{ }^{1} \sigma>\neq 0$ (this can be a process of quantization, or via re-definition of the generating functions in MGTs), we can obtain a dynamical breaking of local supersymmetry with a distorted effective mass for the gravitino,

$$
{ }^{\sigma} \mu=\kappa \sqrt{\frac{11}{2}} \sigma_{c}=\kappa \sqrt{\frac{11}{2}}\left({ }_{0} \sigma_{c}+{ }_{1} \sigma_{c}\right)={ }_{0}^{\sigma} \mu+{ }_{1}^{\sigma} \mu
$$

where ${ }_{0}^{\sigma} \mu$ is determined from the computations with $\nabla$ and ${ }_{1}^{\sigma} \mu$ is a distortion mass term determined by $\widehat{\mathbf{D}}$. The terms ${ }_{1} \sigma_{c}$ and ${ }_{1}^{\sigma} \mu$ vanish for holonomic configurations and diagonal Einstein metrics.

Let us analyze the issue of the cosmological constant. We can express the above effective Lagrangian in the form

$$
{ }^{e f f} \mathcal{L}=-\frac{|\mathbf{e}|}{2 \kappa^{2}}\left[\widehat{\mathbf{R}}\left(\mathbf{e}_{\beta}\right)-2 \Lambda_{0}+\check{\Lambda}\right]+\ldots
$$

where $\Lambda_{0}+\check{\Lambda}:=\kappa^{2}\left(\sigma_{c}^{2}-f^{2}\right)$ can be identified with a tree-level cosmological constant. For off-diagonal configurations in GR and/or MGTs, we have additional possibilities to consider when $\kappa^{2}\left({ }^{1} \sigma_{c}\right)=\check{\Lambda}$, see (22), if we do not involve quantum corrections. We conclude that even the de Sitter spacetime may not be included into the SG effective Lagrangian ${ }^{s g} \mathcal{L}(566)$, such an effective constant may have two sources: It may be a solution of a quantum effective action (including fluctuations of the metric and gravitino and ghost fields) and/or as a result of nonholonomic off-diagonal interactions of the gravitational and (effective) matter fields.

\section{One-Loop Partition Functions with MGT De- formations}

Let us explain how the method of computing the one-loop effective action elaborated in section IV of [17] and which can be generalized to include off-diagonal terms with the possibility of encoding the MGTs effects. There are three key issues: 1) Expanding about a classical background $\mathbf{g}_{\mu \nu}$ (it can be a standard Euclidean $d S_{4}$ metric), we can study small off-diagonal deformations $\tilde{\mathbf{g}}_{\mu \nu} \rightarrow \mathbf{g}_{\mu \nu}+\zeta_{\mu \nu}$ considering that such deformations are computed both as small noholonomic deformations and constrained fluctuations 
of the action up to quadratic order while working with a small $\zeta_{\mu \nu}$ in a oneloop formalism. 2) The off-diagonal contributions caan also be included in the distortion tensors $\widehat{\mathbf{Z}}$. 3) Finally, a very important mechanism that takes into account possible MGT effects is to consider the effective cosmological constants determined by re-definitions of the generating functions.

Our strategy is to decompose all the physical and ghost fields defined on small off-diagonally deformed background geometries and work with the data $\left(\zeta_{\mu \nu}, \widehat{\mathbf{D}}, \Lambda_{0}+\check{\Lambda}\right)$. In the presence of fermions, we shall use a $\mathrm{N}$-adapted vierbein formalism and the canonical spin $\mathrm{d}$-connection $\widehat{\mathcal{D}}$ which should reproduce the same results as in the metric formalism (see similar constructions in [23] but for LC configurations). We assume also that the one-loop effective action encoding the MGTs contributions can obtained by expanding about a de Sitter background with a renormalized cosmological constant $\Lambda>0$ and to consider that in the limit $\Lambda \rightarrow 0$ the gravitino mass reaches its physical value. The relation between $\Lambda_{0}, \check{\Lambda}$ and $\Lambda$ will be studied below and which will impose certain conditions on the generating parameter $\check{\Lambda}$.

\subsection{Nonholonomic bosonic configurations}

The MGTs contributions will be encoded into the "boldface" operators when the coefficients of the geometric objects are computed with respect to the N-adapted frames.

\subsubsection{Bosonic operators}

Introducing $\zeta_{\mu \nu}=\bar{\zeta}_{\mu \nu}+\mathbf{g}_{\mu \nu} \zeta / 4$, for $\zeta=\mathbf{g}^{\mu \nu} \zeta_{\mu \nu}$, we can apply to eq-(60) the variational formalism elaborated in [16, 17]. Up to quadratic order in $\zeta_{\mu \nu}$, we obtain 7 the effective action

$$
\left.\frac{1}{4 \kappa^{2}} \int d^{4} u \sqrt{|\mathbf{g}|} \mid \frac{1}{2} \bar{\zeta}_{\mu \nu}\left(-\widehat{\mathbf{D}}_{\mu} \widehat{\mathbf{D}}^{\mu}+{ }^{1} \lambda\right) \bar{\zeta}^{\mu \nu}-\frac{1}{2} \zeta\left(-\widehat{\mathbf{D}}_{\mu} \widehat{\mathbf{D}}^{\mu}+{ }^{2} \lambda\right) \zeta-\left(\widehat{\mathbf{D}}^{\mu} \bar{\zeta}_{\mu \nu}-\frac{1}{4} \widehat{\mathbf{D}}_{\nu}\right)^{2} \zeta\right]
$$

where

$$
{ }^{1} \lambda=\frac{13}{2} \Lambda-\frac{3}{2}\left(\Lambda_{0}+\check{\Lambda}\right) \text { and }{ }^{2} \lambda=\frac{5}{2}\left(\Lambda_{0}+\check{\Lambda}\right)-\frac{1}{2} \Lambda .
$$

Our goal is to compute the spectra of the three nonholonomic bosonic operators:

$$
\begin{aligned}
{ }^{0} \widehat{\triangle}(X) \varphi & =\left(-\widehat{\mathbf{D}}_{\mu} \widehat{\mathbf{D}}^{\mu}+X\right) \varphi,{ }^{1} \widehat{\triangle}^{\mu \nu}(X) \xi_{\nu}^{\perp}=\left(-\widehat{\mathbf{D}}^{\mu \nu}+\mathrm{g}^{\mu \nu} X\right) \xi_{\nu}^{\perp} \\
{ }^{2} \widehat{\triangle}^{\mu \nu}(X) \bar{\zeta}_{\mu \nu}^{\perp} & =\left(-\widehat{\mathbf{D}}^{\mu \nu}{ }_{\alpha \beta}+\delta_{\alpha}^{\mu} \delta_{\beta}^{\nu} X\right) \bar{\zeta}_{\mu \nu}^{\perp}
\end{aligned}
$$

\footnotetext{
${ }^{7}$ we omit details on the second variations in the $\mathrm{N}$-adapted formalism etc; the formulas for the transformation of the $\mathrm{N}$-adapted bases into the coordinate-frame ones, when the off-diagonal decompositions are small
} 
were the operators $\widehat{\mathbf{D}}^{\mu \nu}$ and $\widehat{\mathbf{D}}^{\mu \nu}{ }_{\alpha \beta}$ are constructed in a similar form to those presented in [16] but for $\nabla^{\mu} \rightarrow \widehat{\mathbf{D}}^{\mu}$. In above formulas, $X$ is a constant and the "transverse traceless' decompositions are performed in the form:

$$
\begin{aligned}
\mathbf{Y}_{\mu} & =\mathbf{Y}_{\mu}^{\perp}+\widehat{\mathbf{D}}_{\mu} \varphi, \widehat{\mathbf{D}}^{\mu} \mathbf{Y}_{\mu}^{\perp}=0, \mathcal{D} V=\mathcal{D} V^{\perp} \mathcal{D} \varphi \sqrt{\operatorname{det}{ }^{0} \widehat{\triangle}(0)} \\
\mathbf{g}^{\mu \nu} \bar{\zeta}_{\mu \nu} & =0, \widehat{\mathbf{D}}^{\mu} \bar{\zeta}_{\mu \nu}^{\perp}=0, \bar{\zeta}_{\mu \nu}=\bar{\zeta}_{\mu \nu}^{\perp}+2 \widehat{\mathbf{D}}_{(\mu} \xi_{\nu)}^{\perp}+\widehat{\mathbf{D}}_{\mu \nu} \chi-\frac{1}{4} \mathbf{g}_{\mu \nu} \widehat{\mathbf{D}}_{\mu} \widehat{\mathbf{D}}^{\mu} \chi \\
\mathcal{D} \bar{\zeta}_{\mu \nu} & =\mathcal{D} \bar{\zeta}^{\perp} \mathcal{D} \xi_{\nu}^{\perp} \mathcal{D} \chi\left[\operatorname{det}{ }^{1} \widehat{\triangle}^{\mu \nu}(-\Lambda)\right]^{1 / 2} \otimes{ }^{0} \widehat{\triangle}\left(-\frac{4}{3} \Lambda\right) \otimes{ }^{0} \widehat{\triangle}(0)
\end{aligned}
$$

Introducing the distortions $\widehat{\mathbf{D}}=\nabla+\widehat{\mathbf{Z}}$ in the above formulas, we can compute the distortions of operators in a similar fashion as the ones for the LC-configurations. For instance, ${ }^{0} \widehat{\triangle}={ }_{\nabla}^{0} \triangle+{ }_{Z}^{0} \widehat{\triangle}$ etc.

\subsection{2 $\mathrm{N}$-adapted gauge fixing and physical gauge}

If an $\mathrm{N}$-connection splitting is prescribed, both operators $\widehat{\mathbf{D}}$ and $\nabla$ can be used to preserve the symmetries of local Lorentz and infinitesimal coordinate transformations. The first type of symmetries can be fixed by setting the antisymmetric part of $\mathrm{N}$-elongated vielbein to be zero (such a procedure is used in [3]). Adding the standard two parameters, $\alpha$ and $\beta, a \mathrm{~d}-$ covariant gauge fixing term, we can fix the coordinate gauge transformations for the MGTs off-diagonal deformations that are encoded into the bosonic part of the action $S_{B}$ (we write $g f$ as a left label for gauge fixing),

$$
{ }^{g f} S_{B}=-\frac{1}{4 \kappa^{2} \alpha} \int d^{4} u \sqrt{|\mathbf{g}|}\left(\widehat{\mathbf{D}}^{\mu} \zeta_{\mu \nu}-\frac{1+\beta}{4} \widehat{\mathbf{D}}_{\nu} \zeta\right)
$$

It is necessary to introduce a nonholonomic ghost (gh) action for some anticommuting complex d-vector field $\mathbf{C}^{\mu}$ when

${ }^{g h} S_{B}=-\frac{1}{4 \kappa^{2} \alpha} \int d^{4} u \sqrt{|\mathbf{g}|} \overline{\mathbf{C}}^{\mu}\left[-\left(\widehat{\mathbf{D}}_{\mu} \widehat{\mathbf{D}}^{\mu}+\Lambda\right) \delta_{\mu \nu}+\frac{\beta-1}{4}\left(\widehat{\mathbf{D}}_{\mu} \widehat{\mathbf{D}}_{\nu}+\widehat{\mathbf{D}}_{\nu} \widehat{\mathbf{D}}_{\mu}\right)\right] \mathbf{C}^{\nu}$

Integrating this formula and absorbing pre-factors into the normalization of the functional measure, we obtain the ghost partition function,

$$
{ }^{g h} \mathbb{Z}_{B}=\operatorname{det}{ }^{1} \widehat{\triangle}^{\mu \nu}(-\Lambda) \otimes{ }^{0} \widehat{\triangle}\left(\frac{4}{\beta-3} \Lambda\right)
$$

The issue of gauge fixing is addressed in the literature in an alternative way via a "physical" gauge. Let us briefly show how such constructions can 
be performed using $\widehat{\mathbf{D}}$ instead of $\nabla$. Considering $\alpha \rightarrow 0$ in (64), we have to impose the condition

$$
\widehat{\mathbf{D}}_{\mu} \zeta^{\mu \nu}-\frac{1+\beta}{4} \widehat{\mathbf{D}}^{\nu} \zeta=0
$$

Using the projections (63) for $\beta=0$, such conditions can be written as a Killing condition,

$$
\widehat{\mathbf{D}}_{\mu} \widehat{\mathbf{D}}^{\mu} \xi_{\nu}+\widehat{\mathbf{D}}_{\mu} \widehat{\mathbf{D}}_{\nu} \xi^{\mu}=0
$$

for $\xi_{\nu}=\xi_{\nu}^{T}+\widehat{\mathbf{D}}_{\mu} \chi$. Hence, when one incoporates the conditions associated with the MGTs effects, we can impose the 'physical' gauge conditions $\xi_{\nu}=0$.

\subsubsection{MGT partition function}

We sahll follow a general-gauge calculation with the d-operators (62). Similarly to formula (37) in [17], 8 we express the scalar part of the action determined by $\widehat{\mathbf{D}}$ in matrix form,

$$
\frac{1}{4 \kappa^{2}} \int d^{4} u \sqrt{|\mathbf{g}|}\left[\left(\begin{array}{ll}
\zeta & \chi
\end{array}\right)\left(\begin{array}{cc}
\mathbf{A}_{1} & \mathbf{B} \\
\mathbf{B} & \mathbf{A}_{2}
\end{array}\right)\left(\begin{array}{l}
\zeta \\
\chi
\end{array}\right)\right]
$$

where boldface matrix elements are used for geometric d-objects with a canonical distortion. Integrating with respect to the fields, and up to an irrelevant multiplicative factor, we find the bosonic partition function encoding the MGT,

$$
\begin{aligned}
\mathbb{Z}_{B}= & { }^{g h} \mathbb{Z}_{B} \otimes\left(\frac{\operatorname{det}{ }^{0} \widehat{\triangle}\left(-\frac{4}{3} \Lambda\right) \otimes{ }^{0} \widehat{\triangle}(0)}{\operatorname{det}{ }^{2} \widehat{\triangle}\left[{ }^{1} \lambda\right] \otimes{ }^{1} \widehat{\triangle}\left[\alpha\left(\frac{2}{3} \Lambda-{ }^{1} \lambda\right)-\Lambda\right] \otimes\left(\mathbf{A}_{1} \mathbf{A}_{2}-\mathbf{B}^{2}\right)}\right)^{1 / 2} \\
= & \operatorname{det}{ }^{1} \widehat{\triangle}[-\Lambda] \otimes{ }^{0} \widehat{\triangle}\left(\frac{4}{\beta-3} \Lambda\right) \otimes \\
& {\left[{ }^{2} \widehat{\triangle}\left[{ }^{1} \lambda\right] \otimes{ }^{1} \widehat{\triangle}\left(\alpha\left(\frac{2}{3} \Lambda-{ }^{1} \lambda\right)-\Lambda\right) \otimes{ }^{0} \widehat{\triangle}\left(\frac{A_{3} \pm \sqrt{A_{4}}}{6(\beta-3)^{2}}\right)\right]^{-1 / 2} }
\end{aligned}
$$

All values in the expression for the $\mathbb{Z}_{B}$ are expressed in terms of $\mathrm{d}$-operators and algebraic combinations of $\alpha, \beta$ and ${ }^{1} \lambda$ and ${ }^{2} \lambda$, see (61),

$$
\begin{aligned}
\mathbf{B}= & -\frac{3}{16}\left(1+\frac{\beta}{\alpha}\right){ }^{0} \widehat{\triangle}(0){ }^{0} \widehat{\triangle}\left(-\frac{4}{3} \Lambda\right), \mathbf{A}_{1}=\frac{1}{16}\left[\left(3+\frac{\beta^{2}}{\alpha}\right) \widehat{\mathbf{D}}_{\mu} \widehat{\mathbf{D}}^{\mu}+2^{2} \lambda\right], \\
\mathbf{A}_{2}= & -\frac{3}{16}\left(1+\frac{3}{\alpha}\right)^{0} \widehat{\triangle}(0){ }^{0} \widehat{\triangle}\left(-\frac{4}{3} \Lambda\right)^{0} \widehat{\triangle}\left(\frac{4(\alpha-3) \Lambda-6 \alpha^{1} \lambda}{3(\alpha+3)}\right), \\
A_{3}= & 4\left(6 \alpha+\beta^{2}+6 \beta-9\right) \Lambda-6\left(3 \alpha+\beta^{2}\right)^{1} \lambda+6(\alpha+3){ }^{2} \lambda, \\
A_{4}= & 4\left[2(6 \alpha+\beta(\beta+6)-9) \Lambda-3\left(3 \alpha+\beta^{2}\right)^{1} \lambda+3(\alpha+3)^{2} \lambda\right]^{2} \\
& +48(\beta-3)^{2}\left[3 \alpha^{1} \lambda-2(\alpha-3) \Lambda{ }^{2} \lambda .\right.
\end{aligned}
$$

\footnotetext{
${ }^{8}$ we follow a different system of notations
} 
The distorted partition function $\mathbb{Z}_{B}(65)$ transforms into a similar one and associated to Einstein gravity in the Landau-DeWitt gauge if $\widehat{\mathbf{D}} \rightarrow \nabla$. This emphasizes the universality of the methods elaborated in [16, 15, 17]. We can work with different metric compatible linear connections defined by the same metric structure via nonholonomic frame transformations and induced torsion. Hereafter, we shall omit detailed proofs of the formulas and solutions to the equations if they can be obtained via distortion relations of the LCconnection $\nabla$ and with respective substitutions for $\widehat{\mathbf{D}}$ in the $\mathrm{N}$-adapted frame calculations.

\subsection{Nonholonomic fermionic configurations}

Let us consider the operator $\widehat{Ð}$, when

$$
-\widehat{\mathrm{Ð}}^{2} \rightarrow-\widehat{\mathcal{D}}^{2}+\widehat{\mathbf{R}} / 4 \ldots
$$

defines the "square" the covariant spin operator $\widehat{\mathcal{D}}$ with $\widehat{\mathbf{R}}(10)$ being the $\mathrm{d}$-curvature scalar. We can perform an Euclideanisation procedure via the transforms $\gamma^{4} \rightarrow i \gamma_{E}^{4}, \gamma^{j} \rightarrow \gamma_{E}^{j}, \mathbf{e}^{4} \rightarrow \mathbf{e}_{E}^{4}, \mathbf{e}^{j} \rightarrow i \mathbf{e}_{E}^{j}$, for $\underline{j}=1,2,3$, and

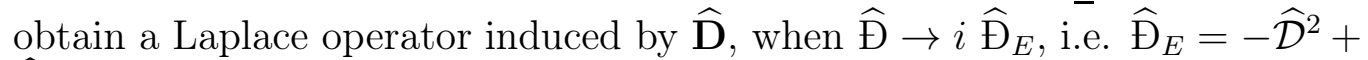
$\widehat{\mathbf{R}} / 4$. Considering $\psi=0$ in the gauge $\psi_{\mu} \gamma^{\mu}=0$, we introduce the standard decompositions

$$
\begin{aligned}
\psi_{\mu} & =\varphi_{\mu}+\frac{1}{4} \gamma_{\mu} \psi, \varphi_{\mu}=\varphi_{\mu}^{\perp}+\left(\widehat{\mathcal{D}}_{\mu}-\frac{1}{4} \gamma_{\mu} \widehat{\mathrm{Ð}}\right) \varsigma \\
\widehat{\mathcal{D}}^{\mu} \varphi_{\mu}^{\perp} & =0, \widehat{\mathcal{D}} \psi_{\mu}=\widehat{\mathcal{D}}^{\mu} \varphi_{\mu}^{\perp} \widehat{\mathcal{D}} \psi \widehat{\mathcal{D}} \varsigma / \sqrt{\operatorname{det} 1 / 2 \widehat{\triangle}\left(-\frac{4}{3} \Lambda\right)},
\end{aligned}
$$

and define the fermionic $\mathrm{d}-$ operators for constant $X$,

$$
{ }^{1 / 2} \widehat{\triangle}(X) \psi=\left(-\widehat{\mathcal{D}}^{2}+\Lambda+X\right) \psi,{ }^{3 / 2} \widehat{\triangle}(X) \varphi_{\mu}^{\perp}=\left(-\widehat{\mathcal{D}}^{2 \mu \nu}+\frac{4}{3} \Lambda \mathbf{g}^{\mu \nu}+X \mathbf{g}^{\mu \nu}\right) \varphi_{\mu}^{\perp} .
$$

The last two terms in ${ }^{\text {eff }} \mathcal{L}(\underline{58})$ can be expressed via $\left(S^{4}\right)$ the use of these $\mathrm{d}-$ operators and a generic mass term $M$,

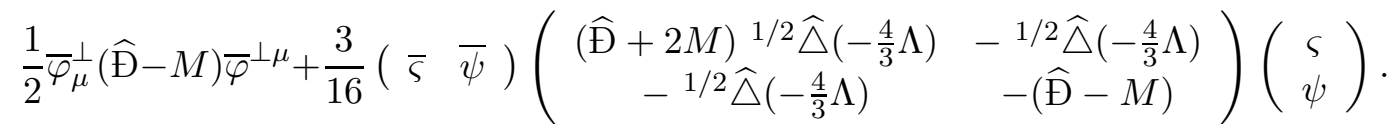

Applying such matrix representations and d-operators (66) (see similar 
details in section IV B of [17]), we compute the gravitino partition function,

$$
\mathbb{Z}_{F}=\left\{\frac{\operatorname{det}^{3 / 2} \widehat{\triangle}\left(\frac{11}{2} \kappa^{2} \sigma_{c}^{2}\right) \otimes{ }^{1 / 2} \widehat{\triangle}\left[\left(\frac{3 \sqrt{2}}{\sqrt{11}} \kappa \sigma_{c}-\frac{8}{11} \sqrt{-\frac{4}{3} \Lambda}\right)^{2}\right]}{\left[\operatorname{det} 1 / 2 \widehat{\triangle}\left(-\frac{4}{3} \Lambda\right)\right]^{3}}\right\}^{1 / 4} .
$$

The coefficients are parameterized as $\sigma_{c}={ }_{0} \sigma_{c}+{ }_{1} \sigma_{c}$ (59) containing contributions from MGT in such a form that similar results are reproduced for $\widehat{\mathrm{D}} \rightarrow \nabla$ and for diagonal configurations in GR.

\subsection{Effective MGT supersymmetry breaking}

We show how possible modifications of gravity theories can contribute to supersymmetry breaking effects in more general supersymmetric models.

\subsubsection{One-loop effective potential and stability}

The distorted partition functions $\mathbb{Z}_{B}$ (65) and $\mathbb{Z}_{F}$ (67) can be used to compute the one-loop effective action

$$
{ }^{1 \text { loop }} \mathcal{S}=-\ln \left(\mathbb{Z}_{B} \otimes \mathbb{Z}_{F}\right)=\frac{1}{2} \ln \operatorname{det}{ }^{2} \widehat{\triangle}\left[{ }^{1} \lambda\right]+\ldots,
$$

following the functional determinant techniques involving the zeta function $\zeta(z):=\sum_{n}^{\infty} g_{n} \lambda_{n}^{-z}$ and derivative $\zeta^{\prime}(0):=-\sum_{n}^{\infty} g_{n} \ln \lambda_{n}$ (see a summary in the Appendix in [17] and Section $\mathrm{V}$ in that paper). A higher energy cut-off is considered via the short time $\epsilon=\mu^{2} / L^{2} \rightarrow 0$ expansion with the constants $\mu$ and $L$ of mass dimension. We can use those formulas by substituting into the functionals the respective functions, $R \rightarrow \widehat{\mathbf{R}}, \Lambda_{0} \rightarrow \Lambda_{0}+\check{\Lambda}$ and $\sigma_{c} \rightarrow \sigma_{c}={ }_{0} \sigma_{c}+{ }_{1} \sigma_{c}$. The finite part of the above functional is expressed as

$$
{ }^{1 \text { loop }} \mathcal{S}=\mathcal{S}_{c}+\left(B_{4}-\mathcal{N}\right) \ln \left(\Lambda / 3 \mu^{2}\right)-B_{4}^{\prime}
$$

for the effective potential

$$
{ }^{\text {eff }} \mathcal{V}=-\frac{\Lambda^{2}}{24 \pi^{2}}{ }^{1 l o o p} \mathcal{S}
$$

where $\mathcal{N}=14-\frac{1}{2} 8=10$ is the number of extra zero modes as elucidated by [16] and $24 \pi^{2} / \Lambda^{2}$ is the spacetime volume for $S^{4}$ of radius $\sqrt{3 / \Lambda}$.

Let us introduce the functions

$$
\zeta_{s}(z, X)=\frac{1}{3}(2 s+1) F\left[z, 2 s+1,(s+1 / 2)^{2}, b_{s}(X)\right],
$$


where $F(z, k, a, b):=\sum_{p=k / 2+1}^{\infty} \frac{p\left(p^{2}-a\right)}{\left(p^{2}-b\right)^{z}}$ and $b_{0}(X)=9 / 4-3 X / \Lambda$, $b_{1 / 2}(X)=-3 X / \Lambda, b_{1}(X)=13 / 4-3 X / \Lambda, b_{3 / 2}(X)=-3 X / \Lambda, b_{2}(X)=17 / 4-$ $3 X / \Lambda$. The coefficients of the action (68) can be computed and expressed as follows:

$$
\begin{aligned}
\mathcal{S}_{c}= & -\frac{1}{2 \kappa^{2}} \int d^{4} u \sqrt{|\mathbf{g}|}\left[\widehat{\mathbf{R}}-2\left(\Lambda_{0}+\check{\Lambda}\right)\right]=-\frac{12 \pi^{2}}{\kappa^{2} \Lambda^{2}}\left(\Lambda_{0}+\check{\Lambda}\right), \\
B_{4}= & \frac{1}{2} \zeta_{2}\left(0,{ }^{1} \lambda\right)-\frac{1}{4} \zeta_{3 / 2}\left(0, \frac{11}{2} \kappa^{2}\left({ }_{0} \sigma_{c}+{ }_{1} \sigma_{c}\right)^{2}\right) \\
& -\zeta_{1}(0,-\Lambda)+\frac{1}{2} \zeta_{1}\left[0, \alpha\left(\frac{2}{3} \Lambda-{ }^{1} \lambda\right)-\Lambda\right] \\
& -\frac{1}{4} \zeta_{3 / 2}\left[0,\left(3 \sqrt{\frac{2}{11}} \kappa\left({ }_{0} \sigma_{c}+{ }_{1} \sigma_{c}\right)-\frac{8}{11} \sqrt{-\frac{4}{3} \Lambda}\right)^{2}\right] \\
& +\frac{3}{4} \zeta_{1 / 2}\left(0,-\frac{4}{3} \Lambda\right)-\zeta_{0}\left(0,-\frac{4}{\beta-3} \Lambda\right)-\frac{1}{2} \zeta_{0}\left(0, \frac{A_{3} \pm \sqrt{A_{4}}}{6(\beta-3)^{2}}\right), \\
B_{4 \mid \Lambda \rightarrow 0}^{\prime} \rightarrow & \frac{6 s+3}{4} \frac{X^{2}}{\Lambda^{2}}\left[\frac{3}{2}-\ln \left(\frac{3 X}{2 \Lambda}\right)\right] .
\end{aligned}
$$

For $\alpha=\beta \rightarrow 0$ and $\Lambda \rightarrow 0$, the data $\mathcal{S}_{c}, B_{4}$ and $B_{4 \mid \Lambda \rightarrow 0}^{\prime}$ from the ${ }^{1 l o o p} \mathcal{S}$ allows to compute the effective distorted potential (the coefficients are taken to be the same as in Section V B of [17], but for additional off-diagonal contributions from the MGTs in ${ }_{1} \sigma_{c}$ ),

$$
\begin{aligned}
e f f \mathcal{V}= & f^{2}-\left({ }_{0} \sigma_{c}+{ }_{1} \sigma_{c}\right)^{2}+\left\{16335 f^{4}-10890\left[f^{2}-32670 f^{2}\left({ }_{0} \sigma_{c}+{ }_{1} \sigma_{c}\right)^{2}\right.\right. \\
& \left.-\left({ }_{0} \sigma_{c}+{ }_{1} \sigma_{c}\right)^{2}\right]^{2} \ln \left[\frac{3 \kappa^{2}}{2 \mu^{2}}\left(f^{2}-\left({ }_{0} \sigma_{c}+{ }_{1} \sigma_{c}\right)^{2}\right)\right]+\left({ }_{0} \sigma_{c}+{ }_{1} \sigma_{c}\right)^{4} \\
& {\left.\left[61156 \ln \frac{\kappa^{2}\left({ }_{0} \sigma_{c}+{ }_{1} \sigma_{c}\right)^{2}}{3 \mu^{2}}-75399+58564 \ln \frac{33}{2}+2592 \ln \frac{54}{11}\right]\right\} . }
\end{aligned}
$$

The term $\ln \left\{\kappa^{2}\left[f^{2}-\left({ }_{0} \sigma_{c}+{ }_{1} \sigma_{c}\right)^{2}\right]\right\}=\ln \left[-\left(\Lambda_{0}+\check{\Lambda}\right)\right]$ may result in an unstable effective potential if $f^{2}<\left({ }_{0} \sigma_{c}+{ }_{1} \sigma_{c}\right)^{2}$. This stability condition imposes a respective limit for ${ }_{1} \sigma_{c}$ and, correspondingly, for $\check{\Lambda}$, i.e. the effective cosmological constant taken from the re-definition of the generating function is related to the stability of the dynamical local breaking of supersymmetry. For certain MGTS and off-diagonal/ nonholonomic configurations, we can obtain stable models and for other ones an instability with an imaginary potential can be obtained. 


\subsubsection{Off-diagonal super-Higgs effects and fermionic and bosonic contributions}

A minimum dynamically generated gravitino mass is computed following the formula (591) when

$$
\begin{aligned}
{ }^{\sigma} \mu & =\kappa \sqrt{\frac{11}{2}}\left({ }_{0} \sigma_{c}+{ }_{1} \sigma_{c}\right)=\sqrt{\frac{11}{16 \pi}} \kappa^{2}\left({ }_{0} \sigma_{c}+{ }_{1} \sigma_{c}\right) M_{P l} \\
& \simeq 1.63730 M_{P l} \simeq 1.99899 \times 10^{19} \mathrm{GeV} .
\end{aligned}
$$

The MGT contributions result in a nontrivial ${ }_{1} \sigma_{c}$ when the global supersymmetry breaking scale is of the order $\sqrt{f} \simeq 0.37876 M_{P l} \simeq 4.62433 \times 10^{18} \mathrm{GeV}$. Such nonholonomic deformations are present at zero and one-loop order and can be separated into bosonic, B, and fermionic, F, parts,

$$
{ }^{e f f} \mathcal{V}=\mathcal{V}_{B}^{(0)}+\mathcal{V}_{B}^{(1)}+\mathcal{V}_{F}^{(1)}, \text { for } \mathcal{V}_{B}^{(0)}=-\kappa^{-2}\left(\Lambda_{0}+\check{\Lambda}\right)
$$

with

$$
\begin{aligned}
\mathcal{V}_{B}^{(1)}= & \frac{45 \kappa^{4}}{512 \pi^{2}}\left[f^{2}-\left({ }_{0} \sigma_{c}+{ }_{1} \sigma_{c}\right)^{2}\right]^{2}\left\{3-2 \ln \left(\frac{3 \kappa^{2}}{2 \mu^{2}}\left[f^{2}-\left({ }_{0} \sigma_{c}+{ }_{1} \sigma_{c}\right)^{2}\right]\right)\right\}, \\
\mathcal{V}_{F}^{(1)}= & \frac{\kappa^{4}\left({ }_{0} \sigma_{c}+{ }_{1} \sigma_{c}\right)^{4}}{30976 \pi^{2}}\left[30578 \ln \frac{\kappa^{2}\left({ }_{0} \sigma_{c}+{ }_{1} \sigma_{c}\right)^{2}}{3 \mu^{2}}\right. \\
& \left.-45867+29282 \ln \frac{33}{2}+1296 \ln \frac{54}{11}\right] .
\end{aligned}
$$

Finally, we note that for the nontrivial minima $\kappa^{4} \mathcal{V}_{F}^{(1)} \simeq-0.791357$ and $\kappa^{4} \mathcal{V}_{B}^{(1)} \simeq 0.0410402$ the tree-level cosmological constant with MGT contributions is constrained to obey the condition

$$
\kappa^{2}\left(\Lambda_{0}+\check{\Lambda}\right) \simeq-0.750279 .
$$

For the LC-configurations, we obtain that the one-loop cosmological constant (vacuum energy) vanishes. This is a result of the stronger spin 2 contributions, and with the opposite sign, the gravitino torsion terms. Nonholonomic torsion terms naturally encode such gravitino contributions via off-diagonal nonlinear interactions. Such a formula is similar to the one for scale symmetry breaking from total derivative densities and in the cosmological constant problem (see [26] and references therein). It can be related to the discovery of the accelerated universe expansion and the "new cosmological constant problem" when the objective is not to explain why it is zero but, instead, it has a very small value for the vacuum energy density. Fixing one of the values $\Lambda_{0}$ and/or $\check{\Lambda}$, in the formula (70), the second one can be considered as a free parameter. 


\subsubsection{On super-Higgs effects for MGT solitonic configurations}

We provided explicit examples of nonlinear solitonic gravitational waves in MGTs in subsection 2.3.4, In general, such solutions contribute to additional terms ${ }_{1}^{\sigma} \mu$ and ${ }_{1} \sigma_{c}$ in (59). For certain special cases like those with generating 2-d solitonic functions of the type $\Phi=Q\left(x^{2}, t\right) s\left(x^{2}, t\right)$ (52), we obtain zero super-Higgs effects if $\Lambda_{0}=0$.

There is a strong correlation between a quantum fluctuation value $\Lambda_{0}$ and an effective source $\check{\Lambda}$ which fix a "scale" for the generating functions via the formulas (42) and (43). For instance, we can chose $\check{\Lambda}$ so that $\mathcal{V}_{B}^{(0)}=$ $-\kappa^{-2}\left(\Lambda_{0}+\check{\Lambda}\right)=0$ in (69) for a computed value of $\Lambda_{0}$. Nevertheless, it is not clear how to satisfy the condition (70) in such a case.

It seems that the super-Higgs effect is a general one which holds true for various classes of off-diagonal configurations determined by GR and/or MGT backgrounds. Certain distinguished backgrounds are possible because for certain configurations $\check{\Lambda}$ has a fixed value determined by (for instance) a configuration which defines a 3-d solitonic hierarchy like in (49). We can change substantially the symmetry of the background solutions, for instance, after introducing a nontrivial $v$-conformal factor resulting in non-Killing symmetries, see (51). Nevertheless, this will not change the fixed values of $\check{\Lambda}$ and the possible quantum fluctuations (via $\check{\omega}^{2}\left(u^{\alpha}\right)$ ) are nonholonomically constrained such that they result in the same $\Lambda_{0}$.

In general, we consider arbitrary solitonic configurations when the effective KP or sine-Gordon equations are not directly involved in the conditions to be solved by the MGT field equations. Dynamical supersymmetry breaking effects are possible because of a nontrivial effective $\check{\Lambda}$ determining a model of nonholonomic Einstein manifolds with induced torsion. But there are also nonholonomic configurations like in (53) when the generating function is fixed by the conditions that MGT field equations are solved for a fixed value of $\check{\Lambda}$. For general solitonic hierarchies, we can select a subset of nonlinear waves which result in a stable effective potential with $f^{2}>\left({ }_{0} \sigma_{c}+{ }_{1} \sigma_{c}\right)^{2}$. It is necessary to have an additional analysis for the "conditional" generating functions if $f^{2}<\left({ }_{0} \sigma_{c}+{ }_{1} \sigma_{c}\right)^{2}$. This can be used to classify off-diagonal solutions and and find if the contributions from MGT are stable or unstable.

\section{Concluding Remarks}

In this article, we studied the problem how modified supergravity theories, MSGTs, have to be formulated to be self-consistent with various classes of modified gravity theories, MGTs, and which were constructed recently in 
connection to modern cosmology and quantum gravity models, the accelerated expansion of the universe, dark energy and dark matter physics. We have chosen for simplicity the specific case of $d=4$ and $\mathcal{N}=1$ supergravity and extended the constructions for off-diagonal configurations with generalized connections. In a certain sense, this is a further development of the paper [17] and early works [16]. On alternative examples of MSGTs, see [18].

We have constructed one-loop effective potentials for various classes of MGTs with nonlinear action functionals, bimetric and massive terms, nonholonomic distributions etc, which can be effectively encoded in off-diagonal Einstein spaces and generalizations with nonholonomically induced torsion. In this way a dynamical mass generation for the gravitino field can be understood in different classes of off-diagonal solutions. We presented certain general illustrative examples when the off-diagonal configurations are described by nonlinear solitonic waves, in three and two dimensions, with one/two Killing and non-Killing symmetries. Due to special kinds of interactions induced by the presence of an effective nonholonomic torsion, the super Higgs effects are naturally computed with respect to nonholonomic frames. In such cases, the gravitational field equations decouple in general forms, and can be integrated also in very general forms and the various quantum fluctuations and off-diagonal nonholonomic deformations can be computed in explicit form. It is possible to define effective nonholonomic operators that mimic (with the use of generalized connections and spin operators) all the calculations elaborated in [17, 16].

We can take into account quantum fluctuations of the generic off-diagonal metrics which were constructed as exact solutions in MGTs. For certain welldefined nonholonomic constraints, the corresponding generating functions can be re-defined and "controlled" by an effective cosmological constant. It is important to impose such constraints and the condition that renormalized constant vanishes only at the end of computations. We can fix a given scale and break the global symmetry which may be stable, or not, depending on the type of MGTs and the chosen class of off-diagonal solutions for the effective Einstein manifolds.

It is essential to choose such value of the effective cosmological constant, when the imaginary parts of the effective action (having proved the existence of the respective vacua) vanish, and attain a stable mechanism for the acquisition of a dynamical mass for the gravitino. To fix the mass to be of the order of the Planck mass in such supergravity models is possible, although additional considerations are necessary in conformal supergravities etc. A rigorous and more complete analysis of such directions to follow and their possible applications in modern cosmology will be the subjects of future works. 
Acknowledgments: The work is partially supported by the Program IDEI, PN-II-ID-PCE-2011-3-0256 and by an associated visiting research position at CERN.

\section{References}

[1] The Nobel Prize in Physics 2013: François Englert and Peter Higgs, http://www.nobelprize.org/nobel_prizes/physics/laureates/2013/

[2] T. Aaltonen et al. Phys. Rev. Lett. 109 (2012) 071804; G. Aad et al. Phys. Lett. B 716 (2012) 1; S. Chatrchyan et al. Phys. Lett., B 716 (2012) 30

[3] P. Van Nieuwenhuizen, Phys. Rept. 68 (1981) 189; H. P. Nilles, Phys. Rept. 110 (1984) 1

[4] Y. Nambu and G. Jona-Lasinio, Phys. Rev. 122 (1961) 345; 124 (1961)

[5] G. Dvali, G. Gabadadze and M. Porrati, Phys. Lett. B 485 (2000) 208; C. Deffayet, G. Dvali and G. Gabadadze, Phys. Rev. D 65 (2002) 044023

[6] S. Vacaru, J. Phys.: Conf. Ser. 543 (2013) 012021; P. Stavrinos and S. Vacaru, Class. Quant. Grav. 30 (2013) 055012; S. Vacaru, Int. J. Geom. Meth. Mod. Phys. 4 (2007) 1285; S. Vacaru, Int. J. Geom. Meth. Mod. Phys. 8 (2011) 9; S. Vacaru, J. Math. Phys. 46 (2005) 042503; S. Vacaru and D. Singleton, Class. Quant. Grav. 19 (2002) 3583; S. Vacaru and D. Singleton, Class. Quant. Grav. 19 (2002) 2793; S. Vacaru and D. Singleton, J. Math. Phys. 43 (2002) 2486-2504; S. Vacaru, JHEP 04 (2001) 009

[7] S. Bazilakos and P. Stavrinos, Phys. Rev. D87 (2013) 043506

[8] S. Vacaru, Int. J. Mod. Phys. D 21 (2012) 1250072; S. Vacaru, Int. J. Geom. Meth. Mod. Phys. 5 (2008) 473; S. Vacaru, Class. Quant. Grav. 30 (2013) 055012; S. Vacaru, Class. Quant. Grav. 28 (2011) 215991; S. Bailakos, N. E. Mavromatos, V. A. Mitsou, and M. Plionis, Astropart. Phys. 36 (2012) 7; A. P. Kouretsis, M. Stathakopoulos and P. C. Stavrinos, Phys. Rev. D86 (2012) 124025; M. Hohmann, Phys. Rev. D87 (2013) 124034; C. Castro, Int. J. Theor. Phys. 51 (2012) 3318; V. A. Kostelecky, N. Russell and R. Tso, Phys. Lett. B716 470; C. Lammerzahl,V. Perlick and W. Hasse, Phys. Rev. D86 (2012) 104042; C. Pfeifer and M. Wohlfarth, Phys. Rev. D84 (2011) 044039 
[9] S. Capozziello and M. De Laurentis, Phys. Rept. 509 (2011) 167; S. Nojiri and S. D. Odintsov, eConf C0602061 (2006) 06 [Int. J. Geom. Meth, Mod. Phys. 4 (2007) 115]; S. Nojiri and S. D. Odintsov, Phys. Rept. 505 (2011) 59; T. Crofton, P. G. Ferreira, A. Padilla and C. Skordis, Phys. Rept. 513 (2012) 1; A. de la Cruz-Dombriz and D. SaezGomez, Entropy 14 (2012) 1717; R. Myrzakulov, L. Seabstiani and S. Zerbini, Int. J. Mod. Phys. D 22 (2013) N8

[10] J. Klusoň, S. Nojiri and S. D. Odintsov, New proposal for non-linear ghost-free massive $F(R)$ gravity: cosmic acceleration and Hamiltonian analysis, arXiv: 1309.2185; S. Vacaru, Off-diagonal ekpyrotic scenaios and equivalence of modified, massive and/or Einstein gravity, arXiv: 1304.1080

[11] S. Vacaru, Generic Off-Diagonal Solutions and Solitonic Hierarchies in Einstein and Modified Gravity, arXiv: 1308.6180; S. Vacaru, OffDiagonal Ekpyrotic Scenarios and Equivalence of Modified, Massive and/or Einstein Gravity, arXiv: 1304.1080; S. Vacaru, Covariant Renormalizable Modified and Massive Gravity Theories on (Non) Commutative Tangent Lorentz Bundles; arXiv: 1304.1079; S. Vacaru, Finsler Spinors and Twistors in Einstein Gravity and Modifications, arXiv: 1206.4012

[12] R. S. Jasinschi and A. W. Smith, Phys. Lett. B 173 (1986) 297; B 174 (1986) 183

[13] S. Deser and B. Zumino, Phys Rev. Lett. 38 (1977) 1433

[14] D. Volkov and V. P. Akulov, Phys. Lett. B 46 (1973) 109; Theor. Math. Phys. 18 (1974) 28 [Teor. Mat. fiz. 18 (1974) 39]

[15] I. L. Buchbinder and S. D. Odintsov, Class. Quant. Grav. 6 (1989) 1955; S. D. Odintsov, Phys. Lett. B. 213 (1988) 7

[16] E. S. Fradkin and A. A. Tseytlin, Nucl. Phys. B 234 (1984) 472

[17] J. Alexandre, N. Houston, N. E. Mavromatos, Dynamical Supergravity Breaking via the Super-Higgs Effect Revisited, arXiv: 1310.4122

[18] S. V. Ketov, Int. J. Mod. Phys. A 28 (2013) 133002; S. V. Ketov and A. A. Starobinsky, JCAP 02 (2012) 022; S. V. Ketov and S. Tsujikawava, Phys. Rev. D 86 (2012) 023529; S. V. Ketov and T. Terada, JHEP 07 (2013) 127 
[19] C. de Rham, Phys. Lett. B 681 (2009) 89; C. de Rham and G. Gabadadze, Phys. Rev. D 82 (2010) 044020; C. de Rham, G. Gabadadze, and A. J. Tolley, Phys. Rev. Lett. 106 (2011) 231101; S. Hassan and R. A. Rosen, Phys. Rev. Lett. 108 (2012) 041101; JHEP 1204 (2012) 2012

[20] T. Kobayashi, S. Siino, M. Yamaguchi and D. Yoshida, Phys. Rev. D 86 (2012) 061505

[21] D. V. Volkov and V. P. Akulov, Phys. Lett. B 46 (1973) 109; Theor. Math. Phys. 18 (1974) 28 [Teor. Mat. Fiz. 18 (1974) 39]; S. Deser and B. Zumino, Phys. Rev. Lett. 38 (1977) 1433

[22] S. Vacaru, Nucl. Phys. B 434 (1997) 590; JHEP, 09 (1998) 011; S. Vacaru and O. Tintareanu-Mircea, Nucl. Phys. B 626 (2002) 239; S. Vacaru and F. C. Popa, Class. Quant. Gravity, 18 (2001) 4921; S. Vacaru and P. Stavrinos, Spinors and Space-Time Anisotropy (Athens University Press, Athens, Greece, 2002), 301 pages, gr-qc/0112028; ) S. Vacaru, J. Math. Phys. 50 (2009) 073503

[23] R. Percacci, M. J. Perry, C. N. Pope and E. Sezgin, Beta functions of topologically massive supergravity, arXiv: 1302.0868

[24] S. Vacaru, Acta Applicandae Mathematicae 110 (2010) 73; JHEP, 04 (2001) 009; S. Vacaru and D. Singleton, J. Math. Phys. 43 (2002) 2486; Class. Quant. Grav. 19 (2002) 2793; 3583

[25] B. B. Kadomtsev and V. I. Petrviashvili, Doklady Akademii Nauk SSS 192 (1970) 753 [in Russian]; Sov. Phys. Dokl. 15 (1970) 539 [English translation]

[26] E. I. Guendelman, H. Nishino and S. Rajpoot, Phys. Lett. B732 (2014) 156 